\title{
Tropical Surface Singularities
}

\author{
Hannah Markwig • Thomas Markwig • \\ Eugenii Shustin
}

Received: 27 June 2011 / Revised: 7 August 2012 / Accepted: 10 August 2012 /

Published online: 5 September 2012

(C) Springer Science+Business Media, LLC 2012

\begin{abstract}
In this paper, we study tropicalizations of singular surfaces in toric threefolds. We completely classify singular tropical surfaces of maximal-dimensional geometric type, show that they can generically have only finitely many singular points, and describe all possible locations of singular points. More precisely, we show that singular points must be either vertices, or generalized midpoints and barycenters of certain faces of singular tropical surfaces, and, in some case, there may be additional metric restrictions to faces of singular tropical surfaces.
\end{abstract}

Keywords Tropical geometry $\cdot$ Singularities $\cdot$ Discriminants $\cdot$ Regular subdivisions of lattice polytopes

\section{Introduction}

This paper studies singularities of tropical surfaces in $\mathbb{R}^{3}$. The question what the analogue of a singularity in the tropical world should be is quite natural to ask and has consequently interested several authors recently $[3,4,10]$. The fact that this question is hard to answer in general makes it even more intriguing. We define a point $p$ in a tropical surface $S$ to be singular if there is an algebraic surface $\tilde{S}$, defined over the

H. Markwig

Fachrichtung Mathematik, Universität des Saarlandes, Postfach 151150, 66041 Saarbrücken,

Germany

e-mail: hannah@math.uni-sb.de

T. Markwig (凶)

TU Kaiserslautern, Erwin-Schrödinger-Straße, 67653 Kaiserslautern, Germany

e-mail: keilen@mathematik.uni-kl.de

E. Shustin

School of Mathematical Sciences, Tel Aviv University, Ramat Aviv, Tel Aviv 69978, Israel

e-mail: shustin@post.tau.ac.il 
Puiseux-series with coefficients in $\mathbb{C}$, whose tropicalization is $S$ and which is singular at a point $\tilde{p} \in \tilde{S}$ that tropicalizes to $p$. Given a non-degenerate lattice polytope $\Delta \in \mathbb{R}^{3}$, consider the family $\operatorname{Sing}(\Delta) \subset \mathbb{P}^{\#\left(\Delta \cap \mathbb{Z}^{3}\right)-1}$ of singular hypersurfaces in the toric threefold defined by $\Delta$ whose defining equations have Newton polytope $\Delta$. We assume that $\Delta$ is non-defective, i.e. that $\operatorname{Sing}(\Delta)$ is a hypersurface in $\mathbb{P}^{\#\left(\Delta \cap \mathbb{Z}^{3}\right)-1}$, defined by a polynomial which is then called the discriminant of $\Delta$. The tropicalization $\operatorname{Trop}(\operatorname{Sing}(\Delta))$ of $\operatorname{Sing}(\Delta)$ has been studied in [3] and is called the tropical discriminant. While a general member of $\operatorname{Sing}(\Delta)$ has exactly one singular point, namely a node, an analogous statement is not true in tropical geometry. The reason is that for a given singular tropical surface, there can be several singular tropical surfaces tropicalizing to it, but such that the respective singular points tropicalize to different points in the tropical surface. Consequently, there are also general tropical surfaces with infinitely many singularities. The subset of singular points of a tropical surface does not seem to have any nice structure, in particular it is not a tropical subvariety. Examples 4.5 and 4.3 of [4] show tropical curves with infinitely many resp. two singular points. We concentrate on singular tropical surfaces of maximal-dimensional geometric type (see Definition 8 in Sect. 2.3 for a precise description). These are the singular tropical surfaces whose parameter space is of the maximal possible dimension equal to $\#\left(\Delta \cap \mathbb{Z}^{3}\right)-2$, which, in particular, equals the dimension of the parameter space of singular algebraic surfaces with Newton polygon $\Delta$. Specifically, such tropical surfaces have only finitely many singular points. We completely classify these singular tropical surfaces and describe possible locations of singular points.

Our study is closely related to [4], which deals with singular tropical hypersurfaces of any dimension. There, a more algebraic point of view is taken however: the main result is the description of tropical singular points in terms of Euler derivatives, i.e. tropical equations are given which a point must satisfy to be singular. We concentrate more on the geometry of singular tropical surfaces.

Our paper can be viewed as a sequel to [10], where we studied tropical plane curves with a singular point. The main result of [10] is the classification of singular tropical curves of maximal-dimensional geometric type. A singular point of a tropical curve of maximal-dimensional geometric type is either a "crossing" of two edges, or a three-valent vertex of multiplicity 3 , or it is a point on an edge $e$ of weight two which has equal distance to the two vertices of $e$ (or which satisfies a similar metric condition, respectively). To derive this result, we used the following methods: we considered the family of algebraic curves in a toric surface with a singularity in a fixed point. This family is defined by linear equations, and so its tropicalization is a Bergman fan which can be described in terms of weight classes of flags of flats of the corresponding matroid $[1,6]$. We studied the possible weight classes and classified the corresponding tropical curves. Fixing a different point in the torus yields a shift of the Bergman fan (see Remarks 3.1 and 3.2 of [10]).

Here, we apply the same methods to the family of algebraic surfaces in a toric threefold with a singularity in a fixed point. While the basic ideas we use are the same as in [10], the classification becomes much more complicated and we have to establish and use various facts about lattice polytopes. Also, we concentrate purely on tropical surfaces with only finitely many singularities (contrary to our classification in the curve case in [10]). Our main result is the classification in Theorem 2. Such a 
classification is not possible in higher dimensions (see Remark 6). Theorem 1 tells us for which tropical surfaces there are only finitely many singularities. For more details and notation, see Sect. 4.

Theorem 1 Let $\Delta \subset \mathbb{R}^{n}$ be a non-degenerate convex lattice polytope and denote by $\mathcal{A}=\Delta \cap \mathbb{Z}^{n}$ the lattice points of $\Delta$. Let $F_{u}(\boldsymbol{x})=\max _{m \in \mathcal{A}}\left\{u_{m}+m \cdot \boldsymbol{x}\right\}, \boldsymbol{x} \in \mathbb{R}^{n}$, define a generic (see Definition 16) singular tropical hypersurface $S$. Assume the dual marked subdivision corresponds to a cone of codimension $c$ in the secondary fan. Then the set of singular points in $S$ is a union of finitely many polyhedra of dimension $c-1$.

In the following classification below, we thus want to restrict to the case $c=1$ of generic tropical surfaces $S$ whose dual marked subdivision corresponds to a cone of codimension 1 in the secondary fan. (Not all cones of codimension 1 in the secondary fan correspond to singular tropical surfaces. We do not give a complete classification but restrict to cones of maximal-dimensional geometric type.) It follows that the dual marked subdivision contains a unique circuit and that every marked polytope in the subdivision which does not contain the circuit is a simplex (see Remark 7). We can conclude from Lemma 3.1 of [4] that every singular point of $S$ is contained in the cell of $S$ dual to the circuit.

In addition, we make the assumption that the tropical surface is of maximaldimensional geometric type (see Definition 8 in Sect. 2.3). In this case, the singular tropical surface uniquely defines a codimension one cone of the secondary fan, and, in the dual marked subdivision, all lattice points of $\Delta$ are marked. Our main result is a complete classification of such singular tropical surfaces and of possible locations of their singular points.

Notice that some codimension 1 cones of the secondary fan do not appear in our classification: these correspond to singular tropical surfaces which are not of maximal-dimensional geometric type. In this case the cone cannot be uniquely restored out of the tropical surface, and the singular locus has positive dimension.

In what follows we will usually consider polytopes only up to integral unimodular affine transformations which we refer to as IUA-equivalence.

Theorem 2 Let $F_{u}=\max _{(i, j, k) \in \mathcal{A}}\left\{u_{(i, j, k)}+i x+j y+k z\right\}$ define a singular tropical surface $S$. We assume that $S$ is generic (see Definition 16) and dual to a marked subdivision $T=\left\{\left(Q_{1}, \mathcal{A}_{1}\right), \ldots,\left(Q_{k}, \mathcal{A}_{k}\right)\right\}$ (see Sect. 2.3) of maximal-dimensional geometric type. Assume the dual subdivision corresponds to a cone of codimension 1 in the secondary fan. Then every marked polytope $\left(Q_{i}, \mathcal{A}_{i}\right)$ in $T$ which does not contain the circuit is a simplex, and $S$ contains only finitely many singular points. Their possible locations and dual polytopes, classified up to IUA-equivalence, are as follows:

(a) If the circuit is of dimension 3 (Cases (A) and (B) in Fig. 1), the dual cell is a vertex $V$ of $S$ and this vertex is the only singular point.

(a.1) Either $V$ is adjacent to six edges and nine 2-dimensional polyhedra. Then the dual polytope is IUA-equivalent to a pentatope with vertices $(0,0,0)$, $(1,0,0),(0,1,0),(0,0,1)$ and $(1, p, q)$ with $p$ and $q$ coprime (Case $(A)$ in Fig. 1, see also Fig. 2). 


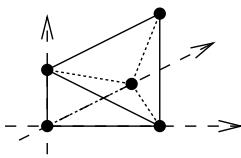

(A)

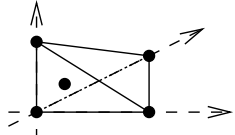

(B)

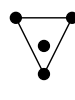

(C)

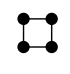

(D)

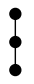

(E)

Fig. 1 The possible circuits

Fig. 2 Case (a.1), a singular tropical surface dual to (A) with the singular point marked

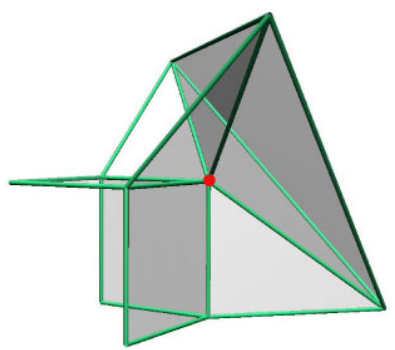

Fig. 3 Case (a.2), a singular tropical surface dual to (B) with the singular point marked

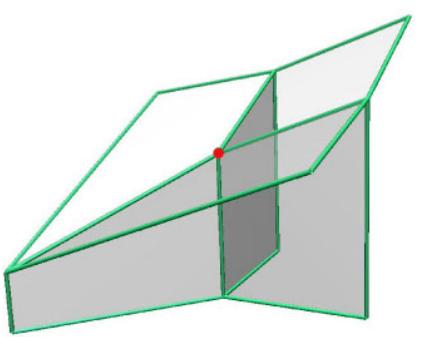

(a.2) $O r V$ is adjacent to four edges and six 2-dimensional polyhedra, just as a smooth vertex (Case (B) in Fig. 1, see also Fig. 3). However, if we define the multiplicity of a vertex of a tropical hypersurface analogously to the case of tropical curves as the lattice volume of the corresponding polytope in the dual subdivision, then it follows that $V$ is a vertex of higher multiplicity. More precisely, the multiplicity can be $4,5,7,11,13,17,19$, or 20. The dual is IUA-equivalent to a tetrahedron with vertices $(0,0,0),(1,0,0),(0,1,0)$ and, resp., $(3,3,4),(2,2,5)$, $(2,4,7),(2,6,11),(2,7,13),(2,9,17),(2,13,19)$, or $(3,7,20)$.

(b) If the circuit is of dimension 2 (Cases $(C)$ and $(D)$ in Fig. 1), the dual cell is an edge $E$. We have the following cases:

(b.1) The dual of $E$ is IUA-equivalent to a triangle with vertices $m_{a}=(0,0,0)$, $m_{c}=(0,1,2)$ and $m_{d}=(0,2,1)$, i.e. $E$ is adjacent to three 2-dimensional cells of $S$ (Case $(C)$ in Fig. 1). Each end vertex of $E$ is adjacent to four edges and six 2-dimensional polyhedra, just as a smooth vertex.

(b.1.1) $E$ is bounded and there is a singularity at the midpoint of $E$ or at points which divide $E$ with the ratio $3: 1$ (see Fig. 4). Or, E is unbounded and there is a singularity whose distance from the vertex of $E$ depends on six coefficients of the tropical polynomial (see Eq. (2) in Sect. 4.3.2). 

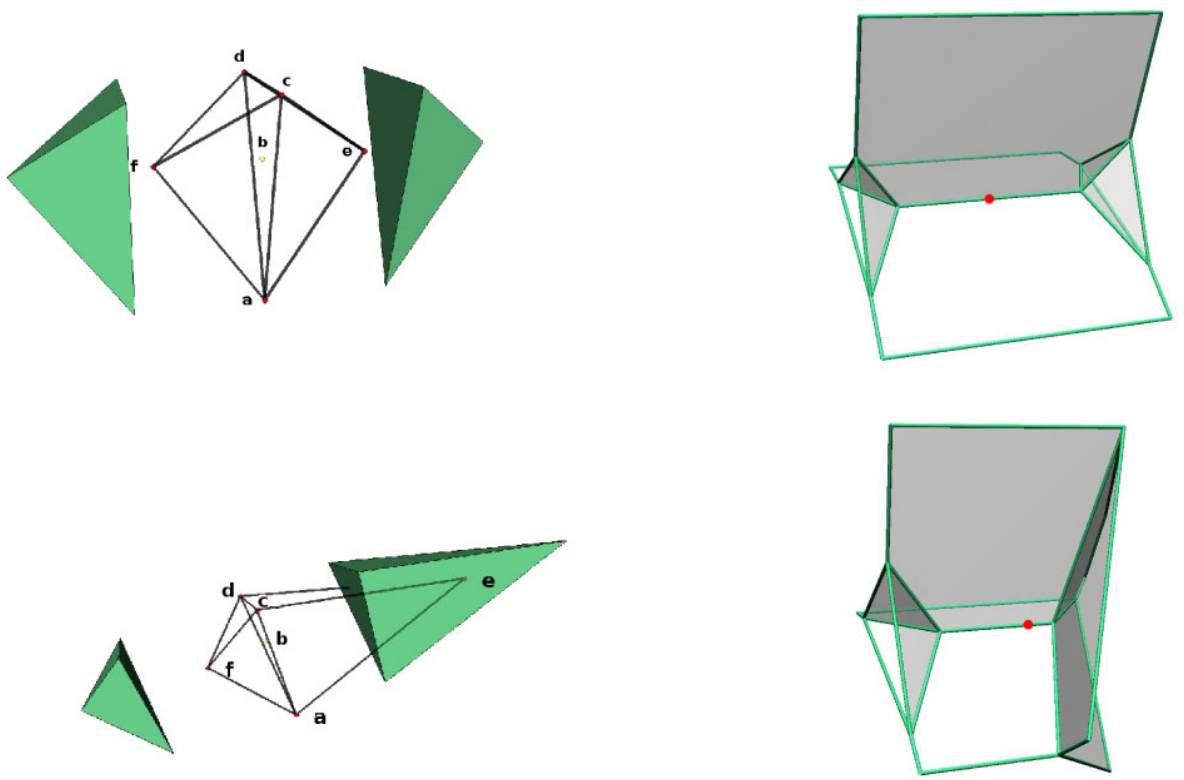

Fig. 4 A singular point which divides the edge $E$ either in the midpoint or with ratio $3: 1$, and dual subdivisions (case (b.1.1))
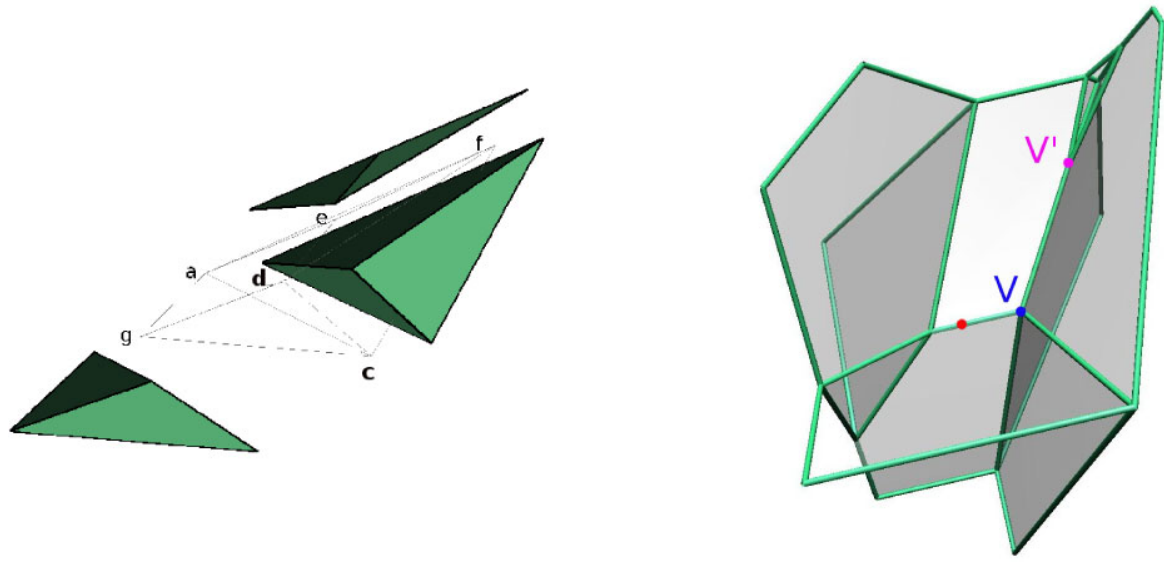

Fig. 5 A tropical surface with a singularity as in case (b.1.2) and its dual subdivision. The edge with the singular point can be extended to an unbounded edge containing only the vertex $V$. The singular point is at distance $d$ from $V$, where $d$ depends on the coefficients involving the two vertices $V$ and $V^{\prime}$

(b.1.2) A bounded edge E admits finitely many (bounded or unbounded) extensions to $a$ virtual edge with a singularity at the positions described in (b.1.1) (see Fig. 5). (Sect. 4.3.2 explains the term virtual edge.) 

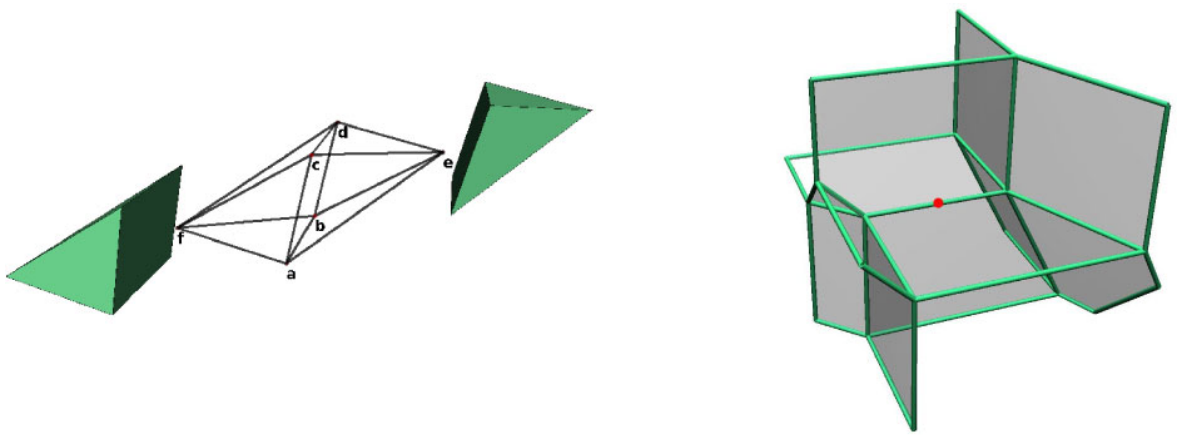

Fig. 6 Case (b.2), a dual subdivision with circuit (D) and the corresponding singular tropical surface with the singular point marked

(b.2) $E$ is dual to a quadrangle, i.e. adjacent to four 2-dimensional cells of $S$ (Case (D) in Fig. 1, see also Fig. 6). E must be bounded and its end vertices are each adjacent to five edges and eight 2-dimensional cells. $S$ contains a unique singular point which is the midpoint of $E$.

(c) If the circuit is of dimension 1 (Case (E) in Fig. 1), then the dual is a 2-dimensional cell of $S$.

(c.1) If this cell is a triangle or trapeze, there is a singular point at the weighted barycenter resp. generalized midpoint, see Sect. 4.5 (for an image see Example 3).

(c.2) An arbitrary 2-dimensional cell admits finitely many extensions to a triangle or a trapeze, with a singularity at the position described in (c.1).

Section 4.5 referred to in statement (c) of Theorem 2 contains a classification of the possible shapes of the cell dual to the circuit and explains the terms weighted barycenter and generalized midpoint.

Example 3 A tropical surface $S$ can have several singularities, since there may be several singular surfaces tropicalizing to $S$ with different images for their singular point. We give here an example for this behavior. Consider the polynomials

$$
\begin{aligned}
f= & \left(1-3 t^{5}-3 t^{8}\right)+\left(-2+t^{5}\right) \cdot z+z^{2}+t^{8} \cdot \frac{1}{x y}+\left(t^{5}+t^{8}\right) \cdot y \\
& +\left(2 t^{5}+t^{8}\right) \cdot x-t^{5} \cdot x^{2} y z
\end{aligned}
$$

and

$$
\begin{aligned}
g= & \left(1-3 t^{6}+3 t^{8}\right)-\left(2+t^{8}\right) \cdot z+z^{2}+t^{8} \cdot \frac{1}{x y}+\left(t^{5}-t^{7}\right) \cdot y \\
& +\left(t^{5}-2 t^{7}\right) \cdot x+t^{5} \cdot x^{2} y z
\end{aligned}
$$

over the field of Puiseux series. They both tropicalize to the tropical polynomial

$$
F_{u}=\max \{0, z, 2 z,-8-x-y,-5+y,-5+x,-5+2 x+y+z\}
$$


Fig. 7 Two singular points on a tropical surface as weighted barycenters

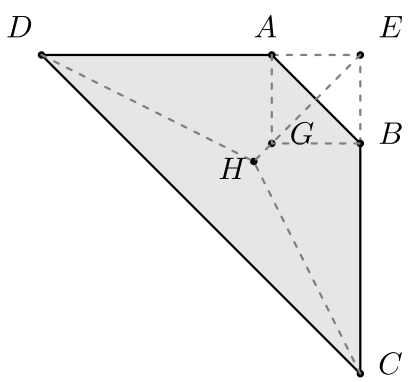

with $u=(0,0,0,-8,-5,-5,-5)$ and define thus the same tropical surface $S$. Moreover, both $V(f)$ and $V(g)$ are singular, however, $V(f)$ is singular in $(1,1,1)$ which tropicalizes to $G=(0,0,0)$, while $V(g)$ is singular in $(t, t, 1)$ which tropicalizes to $H=(-1,-1,0)$. Thus $S$ has two singular points on the quadrangle dual to the circuit formed by $(0,0,0),(0,0,1)$, and $(0,0,2)$. The quadrangle is shown in Fig. 7 , and $G=\frac{1}{3} \cdot(A+B-E)$ and $H=\frac{1}{3} \cdot(C+D+E)$ are weighted barycenters of the vertices $A$ and $B$ respectively $C$ and $D$ with the virtual vertex $E$ in the sense of Theorem 2 and Sect. 4.5 (see also Remark 17 and Examples 22 and 24).

Theorem 2 gives necessary conditions for the geometry of a singular tropical surface. We can also formulate a sufficient condition, which follows from Lemma 10 and the classification:

Theorem 4 Let $F_{u}=\max _{(i, j, k) \in \mathcal{A}}\left\{u_{(i, j, k)}+i x+j y+k z\right\}$ define a tropical surface $S$ dual to a marked subdivision of maximal-dimensional geometric type. Assume that the dual subdivision corresponds to a cone of codimension 1 in the secondary fan, and its unique circuit is one of the IUA-types shown in Fig. 1. Then $S$ is a singular tropical surface if

- either the circuit is of type $(A)$ or $(B)$,

- or the circuit is of type $(C),(D)$, or $(E)$ and it does not lie on the boundary of $\Delta$,

- or the circuit is of type $(C)$, lies on $\partial \Delta$ and it is the base of a pyramid $P$ of the dual subdivision such that $\operatorname{vol}(P)=9$ and $P \varsubsetneqq \Delta$,

- or the circuit is of type $(E)$, lies on $\partial \Delta$, and $\Delta$ contains three more points as described in Proposition 21, or four more points as described in Sect. 4.6.

Furthermore, let $p \in S$ be a point in the cell dual to the circuit, and assume $p$ satisfies conditions (a), (b) or (c) of Theorem 2 above. Then $S$ is the tropicalization of an algebraic surface with a singularity tropicalizing to $p$ if and only if after shifting $S$ such that $p$ becomes the origin (and accordingly adding lineality vectors to the coefficients $u$ such that they become equal along the circuit, see Sect. 3) the flag of subsets $\mathcal{F}(u)$ (see Sect. 2.4) either is a flag satisfying the conditions of Lemma 10, or is in the boundary of such a flag.

Note that Theorems 2 and 4 together give a complete classification of maximaldimensional geometric tropical surfaces and their singular points, and both the necessary and sufficient criteria are easy to verify in any concrete example. 
For circuits of type (C), the singularity condition may impose non-local geometric conditions. Non-local here means that they involve cells of the tropical surface which are not faces of the cell dual to the circuit. The following example presents such a situation.

Example 5 Let us consider the point configuration $\mathcal{A}$ with $m_{a}=(0,0,0), m_{b}=$ $(0,1,1), m_{c}=(0,1,2), m_{d}=(0,2,1), m_{e}=(1,1,1), m_{f}=(3,0,2)$ and $m_{g}=$ $(-1,1,0)$, and a tropical surface $S$ defined by a tropical polynomial $F_{u}, u=$ $\left(u_{a}, u_{b}, \ldots, u_{g}\right)$. We assume that $u_{a}=u_{b}=u_{c}=u_{d} \geq u_{e}, u_{f}, u_{g}$, or equivalently, we assume that the edge $E$ dual to the circuit satisfies $y=z=0$. From Theorem 2 and Sect. 4.3.2 can conclude that $S$ can be singular at the point $p$ which divides $E$ with ratio $3: 1$, or at the point $q$ whose position is determined by Eq. (2), or at the point $r$ with coordinates $\left(\frac{u_{g}-u_{e}}{2}, 0,0\right)$. (The point $r$ is the midpoint of an extension of $E$ to a virtual edge, see Sect. 4.3.2.) For the points $q$ and $r$, the position of the singular point is not locally determined, i.e. it is not determined purely by the linear forms in $F_{u}$ corresponding to the part of the subdivision which is dual to the edge $E$ and its end points, but it involves the vertex $V^{\prime}$ of $S$ determined by the polytope $m_{a}, m_{d}, m_{e}, m_{f}$ (see also Fig. 5).

We now want to specify the sufficient conditions we observe in Theorem 4 in this situation in order to decide which of the points $p, q$ or $r$ is a singular point of the tropical surface. If we move $p$ to the origin, this corresponds to adding the vector $\frac{u_{e}-u_{f}}{2} \cdot(0,0,0,0,1,3,-1)$ to the coefficient vector $\left(u_{a}, u_{b}, u_{c}, u_{d}, u_{e}, u_{f}, u_{g}\right)$. The new coefficients satisfy the conditions of Lemma 10 if and only if the new $g$ coefficient is smaller than the new $e$ and $f$-coefficients which became equal. This is the case if and only if $2 u_{e}>u_{g}+u_{f}$. Moving the point $r$ to the origin corresponds to adding the vector $\frac{u_{g}-u_{e}}{2} \cdot(0,0,0,0,1,3,-1)$. The new coefficients satisfy the conditions if and only if the new $f$-coefficient which equals is smaller than the new $g$ and $e$-coefficients, which again is the case if and only if $2 u_{e}>u_{g}+u_{f}$. Thus $S$ is singular at both points $p$ and $r$ if and only if $2 u_{e} \geq u_{g}+u_{f}$.

Moving the point $q$ to the origin corresponds to adding the vector $\frac{u_{g}-u_{f}}{2}$. $(0,0,0,0,1,3,-1)$ to the coefficient vector. The new coefficient vector satisfies the conditions of Lemma 10 if and only if $2 u_{e}<u_{g}+u_{f}$. Thus $q$ is a singular point of $S$ if and only if $2 u_{e} \leq u_{g}+u_{f}$. If $2 u_{e}=u_{g}+u_{f}$ then $q=r$ and the coefficient vector is in the boundary of three weight classes satisfying the conditions of Lemma 10. In any case, we either have one or two singular points, depending on the coefficients of $u$.

Remark 6 The classification is closely related to the study of $\Delta$-equivalence classes of marked subdivisions (see Sect. 11.3 of [8]), since by Theorem 1.1 of [3], the tropical discriminant (which equals the codimension one subfan of the secondary fan that groups maximal dimensional cones of the secondary fan into $\Delta$-equivalence classes) equals the Minkowski sum of the tropicalization of the family of curves with a singularity in a fixed point and its lineality space. This explains why the dual marked subdivisions of maximal-dimensional geometric singular tropical surfaces correspond to codimension one cones of the secondary fan which separate two non$\Delta$-equivalent maximal cones (see 11.3.10 of [8] for the smooth case): understanding 
the combinatorial types of singular tropical hypersurface is equivalent to understanding $\Delta$-equivalence classes. Since understanding $\Delta$-equivalence classes combinatorially is an open problem for dimension larger than 3 , this connection restricts further generalizations of Theorems 2 and 4 to higher dimensions.

This paper is organized as follows. In Sect. 2 the basic notions will be introduced, most prominently the tropicalization $\operatorname{Trop}(\operatorname{Ker}(A))$ of the family of surfaces in a given toric threefold which are singular at $(1,1,1)$. We also explain how $\operatorname{Trop}(\operatorname{Ker}(A))$ comes in a natural way with a fan structure induced by the matroid associated to $A$, and we describe the full-dimensional cones of this fan as weight classes associated to flags of flats (see Lemma 10). It is well known from [3] that the secondary fan of the point configuration corresponding to $A$ is the Minkowski sum of $\operatorname{Trop}(\operatorname{Ker}(A))$ and the lineality space. In Sect. 3 we reconsider how the Minkowski sum of a cone in $\operatorname{Trop}(\operatorname{Ker}(A))$ with the lineality space can lie in cones of the secondary fan, and we use this to introduce the notion of a generic singular surface as well as to prove Theorem 1. Section 4 is devoted to the classification of generic singular tropical surfaces of maximal-dimensional geometric type, and the classification works along the classification of weight classes in Lemma 10. For the classification also polytopes with certain properties have to be classified, and the corresponding classification results can be found in Sect. 4 too.

\section{Notations and Basic Facts}

In this section, we fix notations and collect basic properties of the family of surfaces with a singularity in a fixed point and its tropicalization, the Bergman fan of the corresponding linear ideal. The content of this section is parallel to Sects. 1, 2 and 3.1 of [10], only now we deal with surfaces instead of curves. We omit proofs in this section, since they are all straightforward generalizations of the corresponding statements in [10].

\subsection{The Family of Surfaces with a Singularity in a Fixed Point}

Fix a non-degenerate convex lattice polytope $\Delta \subset \mathbb{R}^{3}$ and denote by $\mathcal{A}=\Delta \cap \mathbb{Z}^{3}=$ $\left\{m_{1}, \ldots, m_{s}\right\}$ the lattice points of $\Delta$. For any field $\mathbb{K}$ there is a toric threefold $\operatorname{Tor}_{\mathbb{K}}(\Delta)$ associated to $\Delta$ and it comes with the tautological line bundle $\mathcal{L}_{\Delta}$ generated by the global sections $\left\{x^{i} y^{j} z^{k}:(i, j, k) \in \mathcal{A}\right\}$. The torus $\left(\mathbb{K}^{*}\right)^{3}$ is embedded in $\operatorname{Tor}_{\mathbb{K}}(\Delta)$ via

$$
\Psi_{\mathcal{A}}:\left(\mathbb{K}^{*}\right)^{3} \longrightarrow \mathbb{P}_{\mathbb{K}}^{\mathcal{A}}:(x, y, z) \mapsto\left(x^{i} y^{j} z^{k} \mid(i, j, k) \in \mathcal{A}\right)
$$

and inside the torus the elements in the linear system $\left|\mathcal{L}_{\Delta}\right|$ are defined by the equations

$$
f_{a}=\sum_{(i, j, k) \in \mathcal{A}} a_{(i, j, k)} \cdot x^{i} \cdot y^{j} \cdot z^{k}=0
$$


with $a=\left(a_{(i, j, k)} \mid(i, j, k) \in \mathcal{A}\right) \in\left(\mathbb{P}_{K}^{\mathcal{A}}\right)^{*} .\left|\mathcal{L}_{\Delta}\right|$ contains a nonempty linear subsystem $\operatorname{Sing}_{p}(\Delta)$ of surfaces with a singularity at the point $\boldsymbol{p}=(1,1,1)$. The equations for this subsystem are the linear equations

$$
f_{a}(\boldsymbol{p})=0, \quad \frac{\partial f_{a}}{\partial x}(\boldsymbol{p})=0, \quad \frac{\partial f_{a}}{\partial y}(\boldsymbol{p})=0, \quad \frac{\partial f_{a}}{\partial z}(\boldsymbol{p})=0,
$$

or equivalently we can say that the family $\operatorname{Sing}_{p}(\Delta)$ is the kernel of the $4 \times s$ matrix

$$
A=\left(\begin{array}{ccc}
1 & \ldots & 1 \\
m_{1} & \ldots & m_{s}
\end{array}\right) .
$$

Notice that $A$ is just the matrix of the point configuration $\mathcal{A}$, after raising the points to the $\{t=1\}$-plane in $\mathbb{R}^{4}$, if we choose the coordinates $(t, x, y, z)$ on $\mathbb{R}^{4}$.

\subsection{Tropicalizations}

Let $\mathbb{K}$ denote the field of Puiseux series and val the valuation sending a Puiseux series to the smallest exponent. For an ideal $I \subset \mathbb{K}\left[x_{1}^{ \pm}, \ldots, x_{n}^{ \pm}\right]=\mathbb{K}\left[\boldsymbol{x}^{ \pm}\right]$determining a variety $V=V(I) \subset\left(\mathbb{K}^{*}\right)^{n}$ we define the tropicalization of $V$ to be

$$
\operatorname{Trop}(V):=\overline{\left\{\left(-\operatorname{val}\left(x_{1}\right), \ldots,-\operatorname{val}\left(x_{n}\right)\right) \mid\left(x_{1}, \ldots, x_{n}\right) \in V(I)\right\}},
$$

i.e. we map $V$ componentwise with the negative of the valuation map and take the topological closure in $\mathbb{R}^{n}$.

We consider tropicalizations in two situations:

- The tropicalization of $\operatorname{Sing}_{p}(\Delta)=\operatorname{ker}(A)$ : The linear space $V=\operatorname{ker}(A)$ is defined by linear equations over $\mathbb{Q}$. $\operatorname{Trop}(V)$ is the so-called Bergman fan of $I[1,6]$. We will study the Bergman fan $\operatorname{Trop}(\operatorname{ker}(A))$ further in Sect. 2.4. Note that, since the linear generators of $A$ are homogeneous, we will consider $\operatorname{Trop}(V)$ modulo the vector space spanned by $(1, \ldots, 1)$. That is, we consider $\operatorname{Trop}\left(\operatorname{Sing}{ }_{p}(\Delta)\right)=$ $\operatorname{Trop}(\operatorname{Ker}(A))$ as a fan in $\mathbb{R}^{s-1}=\mathbb{R}^{\mathcal{A}} /(1, \ldots, 1)$.

- The tropicalization of a surface $V\left(f_{a}\right)$ with $a \in \operatorname{Sing}_{p}(\Delta)$ : This is an example of a tropical hypersurface. If $V$ is a hypersurface defined by $f=\sum a_{m} \boldsymbol{x}^{m}$, then its tropicalization equals the locus of non-differentiability of the tropical polynomial

$$
\operatorname{trop} f: \mathbb{R}^{n} \longrightarrow \mathbb{R}: \boldsymbol{x} \mapsto \max \left\{-\operatorname{val}\left(a_{m}\right)+m \cdot \boldsymbol{x}\right\}
$$

by Kapranov's Theorem (see [5, Theorem 2.1.1]).

Let us first study the hypersurface case more closely.

\subsection{Tropical Hypersurfaces and Dual Marked Subdivisions}

Tropical hypersurfaces are dual to marked subdivisions $T=\left\{\left(Q_{1}, \mathcal{A}_{1}\right), \ldots\right.$, $\left.\left(Q_{k}, \mathcal{A}_{k}\right)\right\}$ (where the $Q_{i}$ are polytopes and the $\mathcal{A}_{i}$ marked integer points, see [8, Definition 7.2.1] resp. [10, Sect. 2]). We define the type of a marked subdivision to be the subdivision, i.e. the collection of $Q_{i}$ without the markings. 
For a finite subset $\mathcal{A}$ of the lattice $\mathbb{Z}^{d}$ we denote by $\mathbb{R}^{\mathcal{A}}$ the set of vectors indexed by the lattice points in $\mathcal{A}$. A point $u \in \mathbb{R}^{\mathcal{A}}$ induces a regular (or coherent) marked subdivision of $\Delta$ by considering the convex hull of

$$
\left\{\left(m, u_{m}\right) \mid m \in \mathcal{A}\right\} \subset \mathbb{R}^{d} \times \mathbb{R}
$$

in $\mathbb{R}^{d+1}$, and projecting the upper faces onto $\mathbb{R}^{d}$. A lattice point $m$ is marked if the point $\left(m, u_{m}\right)$ is contained in one of the upper faces. We say two points $u$ and $u^{\prime}$ in $\mathbb{R}^{\mathcal{A}}$ are equivalent if and only if they induce the same regular marked subdivision of $\Delta$. This defines an equivalence relation on $\mathbb{R}^{\mathcal{A}}$ whose equivalence classes are the relative interiors of convex cones. The collection of these cones is the secondary fan of $\Delta$.

Regular marked subdivisions of $\Delta$ are dual to tropical hypersurfaces (see e.g. [11, Proposition 3.11]). Given a point $u \in \mathbb{R}^{\mathcal{A}}$ it defines a tropical hypersurface $S_{F}$ as the locus of non-differentiability of the tropical polynomial

$$
F_{u}=\max \left\{u_{m}+m \cdot \boldsymbol{x} \mid m \in \mathcal{A}\right\},
$$

and it defines a regular subdivision of $\Delta$. Each $k$-dimensional polytope in the subdivision is dual to a $d-k$-dimensional orthogonal polyhedron of the tropical hypersurface.

For tropical surfaces dual to a marked subdivision of a polytope in $\mathbb{R}^{3}$, this means more precisely:

- each 3-dimensional polytope in the subdivision is dual to a vertex of the tropical surface;

- each 2-dimensional face in the subdivision is dual to an edge of the tropical surface, which is perpendicular to the plane spanned by the 2-dimensional face;

- each edge of the subdivision is dual to a perpendicular 2-dimensional polyhedron of the tropical surface. The weight of a 2-dimensional polyhedron of the tropical surface is defined to be $\#\left(e \cap \mathbb{Z}^{3}\right)-1$, where $e$ is the dual edge in the marked subdivision.

The duality implies that we can deduce the type of the marked subdivision from the tropical hypersurface $S_{F}$, but not the markings. To deduce the markings, we need to know the coefficients $u_{m}$.

Obviously, the vector $(1, \ldots, 1)$ is contained in the lineality space of the secondary fan. Therefore we can mod out this vector and consider the resulting fan in $\mathbb{R}^{s-1}=$ $\mathbb{R}^{\mathcal{A}} /(1, \ldots, 1)$ with $s=\# \mathcal{A}$. We have seen above that every point $u$ in $\mathbb{R}^{\mathcal{A}}$ defines a tropical hypersurface via the tropical polynomial $F_{u}=\max \left\{u_{m}+m \cdot \boldsymbol{x}\right\}$. Of course, adding 1 to each coefficient $u_{m}$ does not change the tropical hypersurface associated to this polynomial. Hence if we consider $\mathbb{R}^{\mathcal{A}}$ as a parametrising space for tropical hypersurfaces, it makes sense to mod out the linear space spanned by $(1, \ldots, 1)$, and we will do so in what follows. By abuse of notation, we call the fan in $\mathbb{R}^{s-1}$ that we get from the secondary fan in this way also the secondary fan.

The identification of $\mathbb{R}^{\mathcal{A}}$ with $\mathbb{R}^{s}, s=\# \mathcal{A}$, is done by fixing an ordering of the elements of $\mathcal{A}$, say $m_{1}, \ldots, m_{s}$. When referring to an element $u \in \mathbb{R}^{\mathcal{A}}=\mathbb{R}^{s}$ we will sometimes refer to the coordinates of $u$ as $u_{m}$ with $m \in \mathcal{A}$ and sometimes simply as $u_{i}$ with $i=1, \ldots, s$. This should not lead to any ambiguity. 


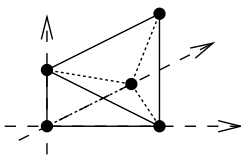

(A)

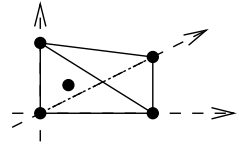

(B)

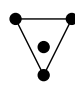

(C)

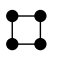

(D)

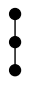

(E)

Fig. 8 Circuits in 3-space

Remark 7 A cone in the secondary fan of codimension one contains exactly one circuit, i.e. a set of lattice points that is affinely dependent but such that each proper subset is affinely independent. A circuit in 3-space consists either of the five vertices of a pentatope such that each subset of four vertices spans the space (A), or of the four vertices of a simplex and an interior point (B), or of four points in a plane as in (C) resp. (D), or of three points on a line (E), as depicted in Fig. 8.

Definition 8 Given a tropical surface $S$, we have seen above that it is dual to a type $\alpha=\left\{Q_{1}, \ldots, Q_{k}\right\}$ of a marked subdivision. We call $\alpha$ also the type of the tropical surface. We can parametrize all tropical surfaces of a given type by an unbounded polyhedron in $\mathbb{R}^{3 \cdot v}$, where $v$ denotes the number of vertices of $S$. We associate a point in $\mathbb{R}^{3 \cdot v}$ to a tropical surface by collecting all coordinates of vertices. The polyhedron is defined by equations and inequalities that we can deduce from the type and that tell us which vertices are connected by an edge of which direction. We define the dimension $\operatorname{dim}(\alpha)$ of a type $\alpha$ to be the dimension of this parametrising polyhedron. If the tropical surface $S$ is singular and $\operatorname{dim}(\alpha)=\#\left(\Delta \cap \mathbb{Z}^{3}\right)-2$, which is the maximal possible value for singular tropical surfaces with Newton polytope $\Delta$, we say that $S$ is of maximal-dimensional geometric type.

For the following lemma recall that we consider the secondary fan of $\Delta$ as a fan in $\mathbb{R}^{\mathcal{A}} /(1, \ldots, 1)$.

Lemma 9 Given a marked subdivision $T=\left\{\left(Q_{l}, \mathcal{A}_{l}\right)\right\}$ of $\Delta$ of type $\alpha$, we have

$$
\operatorname{dim}(\alpha) \leq \operatorname{dim}\left(C_{T}\right)
$$

where $C_{T}$ denotes the cone of the secondary fan corresponding to $T$. Equality holds if and only if in $T$ all lattice points of $\Delta$ are marked, i.e. if $\bigcup_{l} \mathcal{A}_{l}=\Delta \cap \mathbb{Z}^{3}$.

The proof is analogous to Lemma 2.5 of [10].

Since many tropical polynomials can induce the same tropical surface, the secondary fan is not the parameter space for tropical surfaces. However, singular tropical surfaces of maximal-dimensional geometric type are parametrized by the union of (the interior of) codimension one cones of the secondary fan which correspond to dual marked subdivisions with all lattice points marked. This feature also explains our interest in singular tropical surfaces of maximal-dimensional geometric type. 


\subsection{The Tropicalization of $\operatorname{Sing}_{p}(\Delta)=\operatorname{ker}(A)$}

We use the following known results about the tropicalization of linear spaces ([17], Sect. 9.3, $[1,6])$. The tropicalization of the linear space $\operatorname{ker}(A)$ depends only on the matroid $M$ associated to $A$ as follows: we define $M$ by its collection of circuits, which are minimal sets $\left\{i_{1}, \ldots, i_{r}\right\} \subset\{1, \ldots, s\}$ such that the columns $b_{i_{1}}, \ldots, b_{i_{r}}$ of a Gale dual $B$ of $A$ are linearly dependent. A Gale dual is a matrix $B$ whose rows span the kernel of $A$. Given $u \in \mathbb{R}^{s}$, let $\mathcal{F}(u)$ denote the unique flag of subsets

$$
\emptyset=: F_{0} \varsubsetneqq F_{1} \varsubsetneqq \cdots \varsubsetneqq F_{k} \varsubsetneqq F_{k+1}:=\{1, \ldots, s\}
$$

such that

$$
u_{i}<u_{j} \Longleftrightarrow \exists l: i \in F_{l-1} \text { and } j \notin F_{l-1} \text {. }
$$

In particular,

$$
u_{i}=u_{j} \Longleftrightarrow \exists l: i, j \in F_{l} \backslash F_{l-1} .
$$

The weight class of a flag $\mathcal{F}$ is the set of all $u$ such that $\mathcal{F}(u)=\mathcal{F}$.

A flag $\mathcal{F}$ is a flag of flats of the Gale dual $B$ of $A$ respectively of the associated matroid $M$ if the linear span of the vectors $\left\{b_{j} \mid j \in F_{i}\right\}$ contains no $b_{k}$ with $k \notin F_{i}$. As before, the vectors $b_{j}$ denote the columns of $B$. It follows from Theorem 1 of [1] resp. Theorem 4.1 of [6] that the Bergman fan of a matroid $M$ is the set of all weight classes of flags of flats of $M$.

As a consequence, we can study $\operatorname{Trop}(\operatorname{ker}(A))$ by studying weight classes of flags of flats of a Gale dual of $A$. Note that since $A$ is a $4 \times s$-matrix, maximal flags of flats can be identified with flags of $s-4$ subspaces $V_{i} \subset \mathbb{R}^{s-4}$ :

$$
\{0\} \varsubsetneqq V_{1} \varsubsetneqq \cdots \varsubsetneqq V_{s-4},
$$

where each $V_{i}$ is generated by a subset of the column vectors $b_{j}$ of the Gale dual $B$ of $A$ indexed by the set $F_{i}$, and the vectors $\left\{b_{j} \mid j \in F_{i}\right\}$ are all the column vectors of the Gale dual that are contained in the subspace $V_{i}$. In particular, $F_{s-4}=\{1, \ldots, s\}$. We set $F_{i}^{\prime}:=F_{i} \backslash F_{i-1}$. Each $F_{i}^{\prime}$ must of course consist of at least one element $j$. Since we have $s$ vectors in total, we have four "extra" vectors that can a priori belong to any of the $F_{i}^{\prime}$. In the next lemma, we show how the four extra vectors can be spread.

Lemma 10 With the notation from above, for each flag of flats $\mathcal{F}=\mathcal{F}(u)$ of a Gale dual $B$ of $A$ we have either

(a) $\# F_{i}^{\prime}=1$ for all $i=1, \ldots, s-5$ and $\# F_{s-4}^{\prime}=5$, or

(b) $\# F_{s-4}^{\prime}=4$ and there is a $j \in\{1, \ldots, s-5\}$ with $\# F_{j}^{\prime}=2$, or

(c) $\# F_{s-4}^{\prime}=3$ and there is a $j \in\{1, \ldots, s-5\}$ with $\# F_{j}^{\prime}=3$, or

(d) $\# F_{s-4}^{\prime}=3$ and there are $i<j \in\{1, \ldots, s-5\}$ with $\# F_{i}^{\prime}=\# F_{j}^{\prime}=2$.

In each case, the lattice points corresponding to the indices in $F_{s-4}^{\prime}$ form a circuit. In the first case, this is a circuit of type $(A)$ or $(B)$ as in Remark 7, in the second case of type $(C)$ or $(D)$, and in the third and fourth case of type $(E)$. 
In the second case, all points $m_{r}$ with $r \in F_{l}^{\prime}, l>j$, are on the same plane as the four points of $F_{s-4}^{\prime}$, and none of the points with $r \in F_{j}^{\prime}$ is on this plane.

In the third case, all points $m_{r}$ with $r \in F_{l}^{\prime}, l>j$, are on the same line as the three points of $F_{s-4}^{\prime}$, and each choice of two of the points in $F_{j}^{\prime}$ spans the space together with the three points of $F_{s-4}^{\prime}$.

In the fourth case, all points $m_{r}$ with $r \in F_{l}^{\prime}, l>j$, are on the same line as the three points of $F_{s-4}^{\prime}$, and all points $m_{r}$ with $r \in F_{l}^{\prime}, j>l>i$, are on the same plane as the three points of $F_{s-4}^{\prime}$ and the two points of $F_{j}^{\prime}$, and the two points of $F_{i}^{\prime}$ do not lie on this plane.

The proof is a straightforward generalization of Lemma 3.7 of [10]. Note that with this Lemma we describe only interior points of cones corresponding to weight classes of top dimension in $\operatorname{Trop}(\operatorname{ker}(A))$. The analogous statement to Remark 3.8 of [10] holds true as well: for any circuit and any choice of points satisfying the affine dependencies as above we can find a corresponding weight class in $\operatorname{Trop}(\operatorname{ker}(A))$. That means that whenever the coefficients of a tropical polynomial meet one of the above conditions, it lifts to a polynomial over $\mathbb{K}$ defining a surface with singularity at $(1,1,1)$.

\section{The Tropical Discriminant Revisited}

For $\boldsymbol{x} \in \mathbb{R}^{n}$ arbitrary, denote by $\boldsymbol{p}_{\boldsymbol{x}} \in\left(\mathbb{K}^{*}\right)^{n}$ a point with $\operatorname{val}\left(\boldsymbol{p}_{\boldsymbol{x}}\right)=\boldsymbol{x}$, and consider the family $\operatorname{Sing}_{\boldsymbol{p}_{\boldsymbol{x}}}(\Delta)$ of surfaces with a singularity in $\boldsymbol{p}_{\boldsymbol{x}}$. Its tropicalization $\operatorname{Trop}\left(\operatorname{Sing}_{\boldsymbol{p}_{\boldsymbol{x}}}(\Delta)\right)$ does not depend on the choice of $\boldsymbol{p}_{\boldsymbol{x}}$. Moreover, it follows from Remark 3.2 of [10] that it is a shift of $\operatorname{Trop}\left(\operatorname{Sing}_{p}(\Delta)\right)=\operatorname{Trop}(\operatorname{ker}(A))$ by a vector which we denote by $v(\boldsymbol{x})$ whose coordinates in $\mathbb{R}^{s} /(1, \ldots, 1)$ are given by the scalar products of the $m \in \mathcal{A}$ with $\boldsymbol{x}$.

If we let $\boldsymbol{x}$ vary over all points in $\mathbb{R}^{n}$, it follows that $v(\boldsymbol{x})$ varies over all points in the rowspace of the matrix $A$ in $\mathbb{R}^{s} /(1, \ldots, 1)$. In the following, we denote the rowspace of $A$ in $\mathbb{R}^{s} /(1, \ldots, 1)$ by $L$. Notice that $L$ also equals the lineality space of the secondary fan.

Notation 11 Let $v: \mathbb{R}^{n} \rightarrow L$ denote the linear map sending $\boldsymbol{x}$ to $v(\boldsymbol{x})=(m \cdot \boldsymbol{x})_{m \in \mathcal{A}}$ as above. Notice that $v$ is a bijective linear map between vector spaces of dimension $n$.

This illustrates the equality $\operatorname{Trop}(\operatorname{ker}(A))+\operatorname{rowspace}(A)=\operatorname{Trop}(\operatorname{Sing}(\Delta))$ which is proved in Theorem 1.1 of [3]. Since we assume that $\Delta$ yields a non-defective point configuration, it follows from [8], 10.1.2, that $\operatorname{Trop}(\operatorname{Sing}(\Delta))$ is a subfan of the codimension-one-skeleton of the secondary fan. Therefore it comes with a natural fan structure given by the secondary fan. Since it equals $\operatorname{Trop}(\operatorname{ker}(A))+\operatorname{rowspace}(A)$, it also comes with a natural fan structure by weight classes of the lattice of flats of the matroid of $A$. In general, these two fan structures are not compatible-cones can overlap, cut through cones, be smashed to lower dimension etc. In the following, we define the notion of a generic tropical surface and restrict our results in Theorems 1 and 2 to generic surfaces - these are surfaces for which the two fan structures locally 
Fig. 9 A subdivision corresponding to a defective weight class

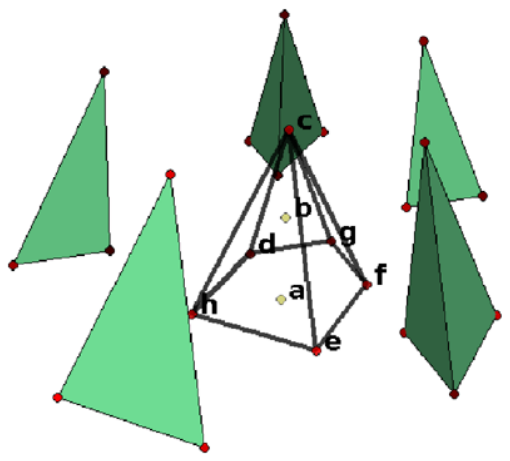

around the coefficient vector $u$ are best compatible. The set of generic surfaces is of top dimension.

Notation 12 For a point $u \in \mathbb{R}^{s} /(1, \ldots, 1)$, we set $C(u)$ the unique cone of the secondary fan with $u \in \operatorname{relint}(C(u))$. Notice that $C(u)=C(u+l)$ for every $l \in L$.

Definition 13 We call a weight class $C$, i.e. a cone of $\operatorname{Trop}(\operatorname{ker}(A))$, defective if there exists a point $u \in C+L$ with $\operatorname{dim}(C+L)<\operatorname{dim}(C(u))$.

Remark 14 If $C$ is a weight class and $u \in C$ such that $C(u)$ has codimension one in the secondary fan, then $C$ is defective if and only if $\operatorname{span}(C) \cap L \neq\{0\}$.

Example 15 We consider the point configuration $\mathcal{A}=\left\{m_{a}, m_{b}, \ldots, m_{h}\right\}$ with

$$
\begin{array}{llll}
m_{a}=(0,0,0), & m_{b}=(0,0,1), & m_{c}=(0,0,2), & m_{d}=(0,1,0), \\
m_{e}=(0,-1,0), & m_{f}=(1,0,0), & m_{g}=(1,1,0), & m_{h}=(-1,0,0)
\end{array}
$$

and we consider the weight class

$$
C=\left\{x_{m_{a}}=x_{m_{b}}=x_{m_{c}}>x_{m_{d}}=x_{m_{e}}>x_{m_{f}}=x_{m_{g}}>x_{m_{h}}\right\} .
$$

The corresponding subdivision of the polytope $\Delta$ is shown in Fig. 9. For a point $u$ in the weight class $C$, the corresponding cone $C(u)$ in the secondary fan is of codimension one. However, the intersection of $\operatorname{span}(C)$ with the lineality space in $\mathbb{R}^{8} /(1, \ldots, 1)$ is 1 -dimensional, since it contains the vector $(0,0,0,0,0,-1,-1,1)$. This shows that the weight class is defective.

Indeed, the weight class $C$ shares a facet with each of the two weight classes

$$
C^{\prime}=\left\{x_{m_{a}}=x_{m_{b}}=x_{m_{c}}>x_{m_{d}}=x_{m_{e}}>x_{m_{f}}=x_{m_{h}}>x_{m_{g}}\right\}
$$

and

$$
C^{\prime \prime}=\left\{x_{m_{a}}=x_{m_{b}}=x_{m_{c}}>x_{m_{d}}=x_{m_{e}}>x_{m_{g}}=x_{m_{h}}>x_{m_{f}}\right\} .
$$


The span of each of these two weight classes intersects the lineality space transversally. The cone $C(u)$ from above is just the union

$$
(C+L) \cup\left(C^{\prime}+L\right) \cup\left(C^{\prime \prime}+L\right),
$$

where actually $C+L$ is not needed, since it is a face of both $C^{\prime}+L$ and $C^{\prime \prime}+L$. This is thus an example that a full-dimensional weight class in $\operatorname{Trop}(\operatorname{Ker}(A))$ may lead to a lower dimensional cone in the tropical discriminant of $A$ which lies in the interior of a full-dimensional cone of the tropical discriminant.

Note that in this example the point configuration $\mathcal{A}$ itself is not defective, however the subset consisting of points $m_{a}, m_{b}, m_{c}, m_{d}, m_{e}, m_{f}$ is.

Assume $C$ is a non-defective weight class, then $C+L$ is contained in cones of the secondary fan of dimension equal to $\operatorname{dim}(C+L)$ or less. The set of all $u \in C$ with $\operatorname{dim}(C+L)>\operatorname{dim}(C(u))$ is obviously of smaller dimension than $\operatorname{dim} C$.

We now define the notion of a generic tropical surface. We choose this definition in such a way that if we decompose the coefficient vector $u$ of a generic surface as $v+l$ with $v \in \operatorname{Trop}(\operatorname{ker}(A))$ and $l \in L$, and $C$ is the weight class of $\operatorname{Trop}(\operatorname{ker}(A))$ containing $v$ in its relative interior, then all dimensions are as expected, i.e. $C$ is of top dimension and $\operatorname{dim}(C+L)=\operatorname{dim} C(u)$.

Definition 16 We call a point $u \in \operatorname{Trop}(\operatorname{Ker}(A))+L \subseteq \mathbb{R}^{s} /(1, \ldots, 1)$ in the tropical discriminant of $\mathcal{A}$ generic if it lies outside the locus formed by $C+L$, where a cone $C$ of $\operatorname{Trop}(\operatorname{Ker}(A))$ either is defective, or is not of the top dimension, or satisfies $\operatorname{dim}(C+L)>\operatorname{dim}(C(v))$. The singular tropical hypersurface defined by the tropical polynomial $F_{u}$ is then also called generic.

From the above, it is obvious that the set of generic points in the tropical discriminant is of top dimension.

Note that in Theorem 2 we consider generic surfaces whose dual marked subdivision is of codimension one in the secondary fan. For defective point configurations, such surfaces do not exist.

Proof of Theorem 1 Let $u \in \operatorname{Trop}(\operatorname{Sing}(\Delta))$ be generic. It follows from the definition of genericity that we can write $u$ as a sum $v+l$ with $v \in \operatorname{Trop}(\operatorname{ker}(A))$ and $l \in L$, such that the weight class $C$ of $\operatorname{Trop}(\operatorname{ker}(A))$ which contains $v$ in its relative interior is top-dimensional and satisfies $\operatorname{dim}(C+L)=\operatorname{dim}(C(v))$. Assume $C(v)=C(u)$ is a cone of codimension $c$ of the secondary fan. Notice that the representation of $u$ as a sum as above is not unique. Firstly, there might be several weight classes $C$ in $\operatorname{Trop}(\operatorname{ker}(A))$ such that we can write $u$ as the sum of a vector in $C$ and a vector in $L$. Secondly, even if we fix one cone $C$, there might be several representations of $u$ as the sum of a vector in this $C$ and a vector in $L$. For now, let us fix one weight class $C$ which allows a representation of $u$ as $u=v+l$ with $v \in C$ and $l \in L$.

Since $\operatorname{dim} \operatorname{Trop}(\operatorname{ker}(A))=s-1-(n+1)$ (where $s=\# \mathcal{A})$ and $v \in C$ is in a top-dimensional weight class, we have $\operatorname{dim}(C+L)=\operatorname{dim}(C)+\operatorname{dim}(L)-$ $\operatorname{dim}(\operatorname{span}(C) \cap L)=s-1-(n+1)+n-\operatorname{dim}(\operatorname{span}(C) \cap L)=\operatorname{dim}(C(v))=$ $s-1-c$, where $\operatorname{span}(C)$ denotes the smallest linear space containing $C$. It follows 
that $\operatorname{dim}(\operatorname{span}(C) \cap L)=c-1$. Therefore there exists a $c-1$-dimensional polyhedron in $H \subset C$ such that for all $h \in H$ we have $v+h \in C$. We can thus write $v$ also as $v=(v+h)-h$, where the first summand is in $C$ and the second summand is in $L$, and these are all possibilities to represent $v$ as a sum of a vector in $C$ plus a vector in $L$. Consequently, we can write $u$ as $u=(v+h)+(l-h)$ and again, these are all possibilities to represent $u$ as a sum of a vector in $C$ and a vector in $L$. It follows that $F_{u}$ defines a tropical surface which is singular at all points $\boldsymbol{x}_{l-h}$, where $\boldsymbol{x}_{l-h} \in \mathbb{R}^{n}$ denotes the preimage of the bijective linear map sending $\boldsymbol{x} \in \mathbb{R}^{n}$ to $v(\boldsymbol{x})=(m \cdot \boldsymbol{x})_{m \in \mathcal{A}}$ from Notation 11. Since the map $v^{-1}$ maps the $c-1$-dimensional polyhedron $l-H$ to a $c-1$-dimensional polyhedron, it follows that all singular points of the surface of $F_{u}$ that we get by decomposing $u$ as a sum of a vector in $C$ and a vector in $L$ lie in a $c-1$-dimensional polyhedron. As we have seen above there may be several (but finitely many) weight classes $C$ in $\operatorname{Trop}(\operatorname{ker}(A))$ such that we can write $u$ as the sum of a vector in $C$ and a vector in $L$, and it thus follows that the set of singular points of the tropical surface defined by $F_{u}$ is a finite union of $c-1$-dimensional polyhedra.

Remark 17 Recall again Example 3 where we had a surface $S$ with two singular points. These two singular points arise because we can interpret the coefficient vector $u$ of the tropical polynomial defining $S$ in two ways as a sum of a vector in a weight class of Trop $(\operatorname{ker}(A))$ and a vector in the lineality space. The two weight classes are different. The point configuration in question corresponds to the matrix

$$
A=\left(\begin{array}{ccccccc}
1 & 1 & 1 & 1 & 1 & 1 & 1 \\
0 & 0 & 0 & -1 & 0 & 1 & 2 \\
0 & 0 & 0 & -1 & 1 & 0 & 1 \\
0 & 1 & 2 & 0 & 0 & 0 & 1
\end{array}\right)
$$

and the singular point $G=(0,0,0)$ on $F_{u}$ with

$$
u=(0,0,0,-8,-5,-5,-5) \in \operatorname{Trop}(\operatorname{Ker}(A))
$$

comes from the weight class containing $u$. However, we can also write $u$ as

$$
u=(0,0,0,-6,-6,-6,-8)+(0,0,0,-2,1,1,3)=v+l,
$$

where

$$
l=(0,0,0,-1,0,1,2)+(0,0,0,-1,1,0,1)=(0,0,0,-2,1,1,3)
$$

belongs to the lineality space of the secondary fan of the point configuration and $v$ belongs to some other weight class. The corresponding singular point on $S$ is $H=(-1,-1,0)$, since we have added once the vector of $x$-coordinates and once the vector of $y$-coordinates to the weight vector $v$ in the weight class in order to get $u$. This corresponds to shifting the whole surface (determined by $F_{v}$, which is singular at 0$)$ by $(-1,-1,0)$ (see also Notation 11 and before). Examples 22 and 24 give further explanations concerning this example.

This shows that even if the point $u$ in the tropical discriminant is generic, the surface corresponding to $u$ may have more than one singular point. 


\section{The Classification}

Now, using the preparation from Sect. 2, we prove Theorem 2. In particular, we consider the points $u \in \operatorname{Trop}(\operatorname{Sing}(\Delta))$ which are generic in the sense of Definition 16, and which in addition satisfy $\operatorname{dim}(C(u))=s-2$, where $C(u)$ is as in Notation 12 . In addition, we work in the situation where the dual marked subdivision as in Sect. 2.3 has all lattice points marked (see Lemma 9). Since we can always write $u=v+l$ for some $a \in \operatorname{Trop}(\operatorname{ker}(A))$ and $l \in \operatorname{rowspace}(A)$, just as in the proof of Theorem 1 above, we can classify the singularities of the tropical surface defined by $F_{v}$ with $v \in \operatorname{Trop}(\operatorname{ker}(A))$ first, and then investigate how the shift to $F_{u}$ effects the location of the singular points. We thus have to consider all different types of weight classes as in Lemma 10, and the corresponding possible types of circuits. It turns out that in most cases we do not have to worry about the shift when passing from $F_{v}$ to $F_{u}$, since we describe the location of the singular point relative to other points in the surface, e.g. as the midpoint of an edge. This midpoint is of course shifted accordingly.

\subsection{Weight Class as in Lemma 10(a), Circuit (A) of Remark 7}

Let $u \in \operatorname{Trop}(\operatorname{ker}(A))$ be in a weight class as in Lemma 10(a), and assume $F_{s-4}^{\prime}=$ $\{a, b, c, d, e\}$. Consider the marked subdivision defined by $u$ as in Sect. 2.3. As the heights of the points $m_{a}, m_{b}, m_{c}, m_{d}$ and $m_{e}$ are biggest, it follows that the convex hull spanned by these points is a polytope of the subdivision. Let us first assume that this polytope is a circuit of type (A) as in Remark 7. The vertex of the tropical surface dual to this pentatope is at the point $(x, y, z)$ where the maximum is attained by the corresponding five terms of trop $\left\{u_{m}+m \cdot(x, y, z)\right\}$, in particular the five terms are equal at this vertex. That means, we can set the five terms equal and solve for $x, y$ and $z$ to get the position of the vertex. But since the coefficients are all equal, we get $x=y=z=0$ when solving. Notice that $(0,0,0)$ is the tropicalization of the singular point $(1,1,1)$.

Since we require that all lattice points are marked, this polytope cannot contain any lattice point besides these five. By Theorem 3.5 of [16], a pentatope which does not contain any lattice point besides its five vertices are IUA-equivalent to the tuple of points $(0,0,0),(1,0,0),(0,1,0),(0,0,1)$ and $(1, p, q)$ with $p$ and $q$ coprime. It is a bipyramid.

It follows that in this situation the node of the tropical surface is at a vertex with six adjacent edges and nine adjacent 2-dimensional polyhedra.

This settles case (a.1) of Theorem 2 .

\subsection{Weight Class as in Lemma 10(a), Circuit (B) of Remark 7}

As above, it follows that the singular point $(0,0,0)$ is dual to the convex hull of $m_{a}, m_{b}, m_{c}, m_{d}$ and $m_{e}$. This is a vertex of the tropical surface with four adjacent edges and six 2-dimensional polyhedra, just as a smooth vertex. However, if we define the multiplicity of a vertex of a tropical hypersurface analogously to the case of tropical curves as the lattice volume of the corresponding polytope in the dual subdivision, then it follows that the singular point is a vertex of higher multiplicity. More precisely, the multiplicity can be $4,5,7,11,13,17,19$ or 20 . This follows 
Fig. 10 The cone $C_{m}$

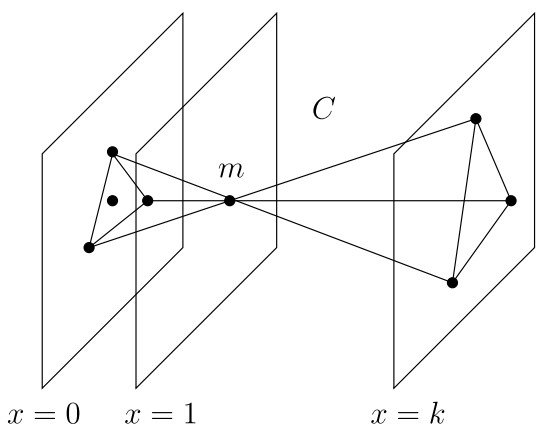

from the classification of 3-dimensional tetrahedra with one interior lattice point (and no other lattice points besides the vertices) (see [15], Theorem 7). Since we require that all lattice points are marked, the tetrahedron which is the convex hull of $m_{a}, m_{b}, m_{c}, m_{d}$ and $m_{e}$ has to be of this form. The classification states that such a tetrahedron is IUA-equivalent to one of the following eight: it has vertices $(0,0,0)$, $(1,0,0),(0,1,0)$ and, respectively, $(3,3,4),(2,2,5),(2,4,7),(2,6,11),(2,7,13)$, $(2,9,17),(2,13,19)$, or $(3,7,20)$. This settles case $($ a. 2$)$ of Theorem 2.

\subsection{Weight Class as in Lemma 10(b), Circuit (C) of Remark 7}

Let $F_{s-4}^{\prime}=\{a, b, c, d\}$ and $F_{j}^{\prime}=\{e, f\}$.

\subsubsection{Assume That in the Subdivision, $m_{e}$ and $m_{f}$ Both Form a Pyramid with the Triangle Spanned by $m_{a}, m_{b}, m_{c}$ and $m_{d}$ as Base}

In particular, $m_{e}$ and $m_{f}$ must lie on different sides of the plane spanned by $m_{a}, m_{b}$, $m_{c}$ and $m_{d}$. Since there are no other circuits, and we require that all lattice points in $\Delta$ are marked, both of these pyramids contain no further lattice points.

Lemma 18 Let four lattice points $m_{a}, m_{b}, m_{c}$ and $m_{d}$ in an affine plane in $\mathbb{R}^{3}$ form a circuit of type $(C)$ as in Remark 7. Let $m_{e}$ be a fifth lattice point that forms a pyramid with this circuit as base and assume this pyramid contains no further lattice points. Then $m_{e}$ has integral distance 1 or 3 from the plane spanned by the circuit.

Proof We can assume that the plane spanned by $m_{a}, m_{b}, m_{c}$ and $m_{d}$ is the $x=0$-plane, and, using a suitable automorphism of $\mathbb{Z}^{2}$, we can bring these four points to $(0,0),(-1,-1),(-2,-1)$ and $(-1,-2)$. Denote the triangle spanned by these points by $T$. Also we assume without restriction that the $x$-coordinate of $m_{e}$ is positive. We have to show that it is then either 1 or 3 . Consider a lattice point $m$ with $x$-coordinate 1 and let $C_{m}$ be the cone with vertex $m$ and spanned by the rays $m, m-(0,-2,-1)$ and $m-(0,-1,-2)$. Intersect this cone with the plane $x=k$ for some choice of $k>1$ (see Fig. 10).

For any lattice point in $C_{m} \cap\{x=k\} \cap \mathbb{Z}^{3}$, consider the pyramid that this point forms with $T$ as base. This pyramid will contain the point $m$. If we move $m$ by a step of integer length 1 , the triangle $C_{m} \cap\{x=k\}$ is shifted by $k$. Compared to $T$ the 
Fig. 11 The shifts of the triangle $C_{m} \cap\{x=k\}$ on the plane $\{x=k\}$

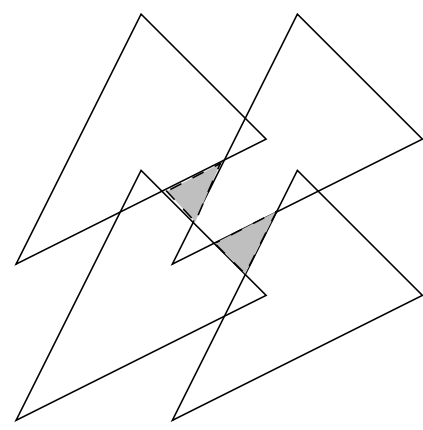

Fig. 12 The non-covered region for different values of $k$

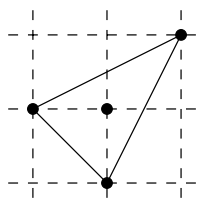

$k \equiv 0 \bmod 3$

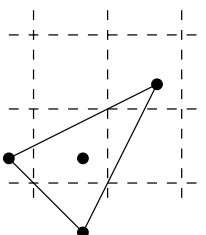

$k \equiv 1 \bmod 3 \quad k \equiv 2 \bmod 3$

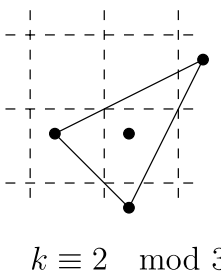

triangle $C_{m} \cap\{x=k\}$ is grown by a factor of $k-1$. Of course, we can also move $m$ to a point with a different $x$-coordinate, this will add more triangles (smaller in size) such that for each point inside a triangle we know that the corresponding pyramid contains another lattice point. We show that for $k \neq 3$ the shifted triangles cover all lattice points with $x$-coordinate $k$. It follows that any pyramid with $T$ as base and with a vertex with $x$-coordinate $k \neq 1,3$ contains another lattice point. Figure 11 shows the plane $\{x=k\}$ with the $k$-shifts of the triangle $C_{m} \cap\{x=k\}$.

Let us compute the vertices of the right shaded region which is not yet covered by a triangle. Assume the left most vertex of the top right triangle has coordinates $(1,1)$ in the plane, then it follows that the coordinates of the vertices of the shaded region are $\left(\frac{2 k}{3}-1, \frac{k}{3}\right),\left(\frac{2 k}{3}+1, \frac{k}{3}+1\right)$ and $\left(\frac{2 k}{3}, \frac{k}{3}-1\right)$. Independently of $k$, this is a triangle of lattice area 3 , with the point $\left(\frac{2 k}{3}, \frac{k}{3}\right)$ as midpoint from which we reach the three vertices by a lattice step to the left, down, and to the upper right. This triangle has an interior lattice point if and only if $k$ is divisible by 3 . In this case, the lattice point is $\left(\frac{2 k}{3}, \frac{k}{3}\right)$ (see Fig. 12).

Analogously, we can compute the vertices of the left shaded region and see that it has an interior lattice point if and only if $k$ is divisible by 3 , and then this lattice point has coordinates $\left(\frac{k}{3}, \frac{2 k}{3}\right)$. It follows that for any $k$ which is not divisible by 3 the $k$-shifts of the triangle $C_{m} \cap\{x=k\} \cap \mathbb{Z}^{3}$ cover already all lattice points. That is, any pyramid with $T$ as base and with a vertex with $x$-coordinate which is not divisible by 3 contains another lattice point with $x$-coordinate 1 .

If $k$ is divisible by 3 , then a pyramid with a vertex with coordinates $\left(k, \frac{2 k}{3}+\right.$ $\left.i k, \frac{k}{3}+j k\right)$ or $\left(k, \frac{k}{3}+i k, \frac{2 k}{3}+j k\right)$ where $i, j \in \mathbb{Z}$ does not contain a lattice point with $x$-coordinate 1 . Here, we take the effect of the $k$-shifts of $C_{m} \cap\{x=k\} \cap \mathbb{Z}^{3}$ on the shaded regions into account. Let us now assume that $k=3^{l} \cdot h$, where $h \neq 1$ and $3 \nmid h$. Now move $m$ to a point with $x$-coordinate $3^{l}$. It follows using the same 
arguments as above that any pyramid with base $T$ and a vertex with $x$-coordinate $k$ contains a lattice point with $x$-coordinate $3^{l}$. Next assume that $k=3^{l}, l \geq 2$. Using $m$ with $x$-coordinate 1 as before we see that the only possibilities to get a pyramid which does contain a lattice point with $x$-coordinate 1 are that the vertex has $(y, z)$ coordinates divisible by $3^{l-1}$ and not by $3^{l}$. Using $m$ with $x$-coordinate 3 we see that the only possibilities to get a pyramid which does not contain a lattice point with $x$-coordinate 3 are that the vertex has $(y, z)$-coordinates divisible by $3^{l-2}$ and not by $3^{l-1}$. As there is no vertex which satisfies both it follows that any pyramid with a vertex with $x$-coordinate $k=3^{l}, l \geq 2$, contains a lattice point with $x$-coordinate 1 , or it contains a lattice point with $x$-coordinate 3 . In any case, it contains another lattice point. It follows that the $x$-coordinate of the vertex $m_{e}$ can only be 1 or 3 .

Remark 19 There are vertices $m_{e}$ with integral distance 1 and 3 to the plane containing the circuit of type (C) of Remark 7 such that the pyramid formed by the circuit and $m_{e}$ contains no further lattice points, e.g. the convex hull of the points $(0,1,0)$, $(0,0,1),(0,2,2)$ and $(3,0,2)$, or the convex hull of the points $(0,0,0),(0,1,2)$, $(0,2,1)$ and $(1,0,0)$.

Now solve the equations given by the tropical polynomial to get the positions of the two vertices corresponding to the two pyramids. The $x$-coordinates of $m_{e}$ and $m_{f}$ can either be the negative of each other, or one can be 3 and the other -1 . Since $m_{a}, m_{b}, m_{c}$ and $m_{d}$ have biggest and equal height, it follows that the edge dual to the convex hull of $m_{a}, m_{b}, m_{c}$ and $m_{d}$ satisfies the equations $y=0$ and $z=0$. If $\lambda=u_{m_{a}}$ is the biggest weight (the weight of $m_{a}, m_{b}, m_{c}$ and $m_{d}$ ), and $\mu=u_{m_{e}}$ is the weight of $m_{e}$ and $m_{f}$, it follows that the vertex dual to the pyramid with vertex $m_{e}$ is at $(\mu-\lambda, 0,0)\left(\operatorname{resp} .\left(\frac{1}{3} \cdot(\mu-\lambda), 0,0\right)\right)$ and the vertex dual to the pyramid with vertex $m_{f}$ is at $(\lambda-\mu, 0,0)$ (resp. $\left.\left(\frac{1}{3} \cdot(\lambda-\mu), 0,0\right)\right)$. It follows that the singular point $(0,0,0)$ is either exactly in the middle of the edge dual to the convex hull of $m_{a}, m_{b}, m_{c}$ and $m_{d}$, or subdivides the edge into parts whose distances have ratio $1: 3$. This explains the first cases of (b.1) in Theorem 2.

\subsubsection{Assume that at Most One of the Points $m_{e}$ and $m_{f}$ Forms a Pyramid with the Triangle Spanned by $m_{a}, m_{b}, m_{c}$ and $m_{d}$ as Base}

As before, assume that $m_{a}=(0,0,0), m_{b}=(0,1,1), m_{c}=(0,2,1)$ and $m_{d}=$ $(0,1,2)$. Any point $m$ that forms a pyramid with the triangle as base must have the absolute value of the $x$-coordinate 1 or 3 due to Lemma 18 , since otherwise we would get extra lattice points, contradicting our assumption that the plane is of maximaldimensional geometric type. If $m$ is a point different from $m_{e}$ and $m_{f}$ but with $x$-coordinate 1 or -1 , then it cannot form a pyramid with the triangle as base since its coefficient is too low. Thus we can conclude without restriction that in this situation, there is a pyramid with the triangle as base and with a vertex $m$ with $x$-coordinate 3 .

Assume that $m_{e}$ does not form a pyramid with the triangle as base. We now determine the possible $x$-coordinates of $m_{e}$. We have seen already that then there is a pyramid with vertex $m$ with $x$-coordinate 3 . Because $\mu=u_{m_{e}}$ is the second biggest coefficient, the $x$-coordinate of $m_{e}$ must be smaller than 3 . It cannot be 2 however, 
since then by Lemma 18 the pyramid formed by $m_{e}$ and the triangle contains further lattice points. Even if this pyramid is not part of the subdivision, these additional lattice points would be contained in the convex body spanned by $m, m_{e}$ and the triangle. Because of their coefficients smaller $\mu$, they cannot be marked points of the subdivision, contradicting our assumption that the surface is of maximal-dimensional geometric type. With the same arguments, $m_{e}$ cannot have $x$-coordinate -2 or smaller -3 . It follows that it must have the absolute value of the $x$-coordinate 1 .

There are a priori several possibilities for weight classes as in Lemma 10(b) from which our subdivision can arise. In order to determine these possibilities, we have to decompose the coefficient vector $u \in \operatorname{Trop}(\operatorname{Sing}(\Delta))$ of our tropical polynomial as a sum $v+l$ where $v$ is in a feasible weight class and $l$ is in the rowspace of $A$. Assume we have already added vectors of the rowspace to $u$ to achieve that the four points of the circuit have equal and biggest coefficients. Next we add a multiple of the vector of $x$-coordinates to make two coefficients of points outside the plane of the circuit equal and second biggest, the two points $m_{e}$ and $m_{f}$. To all points with $x$-coordinate one, we add the same value by adding the multiple of this rowspace vector. Thus there is a unique point with $x$-coordinate \pm 1 which is a candidate to be $m_{e}$ resp. $m_{f}$ - the one with the biggest coefficient after adding rowspace vectors that make the coefficients of the circuit equal. Candidates for $m_{e}$ and $m_{f}$ are now points with $x$-coordinate \pm 3 that form a pyramid with the triangle as base, and points with $x$-coordinate \pm 1 whose coefficient is biggest after adding rowspace vectors to make the coefficients of the circuit equal. Also, $m_{e}$ and $m_{f}$ must have different $x$-coordinates since otherwise the weight class would not intersect the corresponding weight class transversely which contradicts our assumption that $u$ is generic (see Definition 16).

We therefore have the following four possibilities for weight classes (without restriction):

- Let $m$ with $x$-coordinate 3 form a pyramid with the triangle, and let $m_{e}$ be a point with $x$-coordinate one. Let $m_{f}$ with $x$-coordinate -1 form a pyramid with the triangle.

- Let $m$ with $x$-coordinate 3 form a pyramid with the triangle, and let $m_{e}$ be a point with $x$-coordinate one. Let $m_{f}$ with $x$-coordinate -3 form a pyramid with the triangle.

- Let $m_{1}$ with $x$-coordinate 3 and $m_{2}$ with $x$-coordinate -3 form a pyramid with the triangle. Let $m_{e}$ be a point with $x$-coordinate 1 and $m_{f}$ with $x$-coordinate -1 .

- Let $m=m_{f}$ with $x$-coordinate 3 form a pyramid with the triangle, and let $m_{e}$ be a point with $x$-coordinate one.

In each of the four cases, the rowspace of $A$ intersects the corresponding weight class transversely, and so there is at most one solution to decompose $u$ as a sum. The decomposition must be possible in at least one of the cases.

The fourth case has to be treated separately. Note that the fourth case is the only one which can also arise if the edge dual to the triangle is unbounded.

In the first three cases, we introduce the notion of a virtual edge dual to the triangle. This virtual edge is just as the actual edge dual to the triangle contained in the line $y=z=0$, however it ends at points whose $x$-coordinates differ from the actual $x$-coordinates of the vertices dual to the pyramids adjacent to the triangle. For a fixed 
weight class, i.e. for a fixed choice of $m_{e}$ and $m_{f}$ as above, we define the virtual vertex corresponding to $m_{e}$ to be the vertex dual to the pyramid formed by the triangle and $m_{e}$ (even though this pyramid is not part of the subdivision). In the third case, we also define the virtual vertex of $m_{f}$ analogously. The virtual edge connects the virtual vertex of $m_{e}$ with the (virtual or actual) vertex of $m_{f}$. Note that the virtual edge contains the actual edge. It follows from the previous subsection that in the first and third case, the singular point is the midpoint of the virtual edge while in the second case, it subdivides the virtual edge with ration $1: 3$.

Let us treat the first case exemplarily with more details. Denote by $\lambda$ the coefficient of $m_{a}, m_{b}, m_{c}$ and $m_{d}$, by $\mu$ the coefficient of $m_{e}$ and $m_{f}$ and by $v$ the coefficient of the point $m$ which forms a pyramid with the triangle. We have $v<\mu<\lambda$. The virtual vertex of $m_{e}$ has coordinates $(\lambda-\mu, 0,0)$, the actual vertex-i.e. the vertex corresponding to the pyramid formed by $m$ and the triangle- has coordinates $\left(\frac{\lambda-v}{3}, 0,0\right)$. Since $m_{e}$ has $x$-coordinate 1 and $m$ has $x$-coordinate 3 but forms a pyramid with the triangle, we must have $\mu<\frac{2 \lambda+v}{3}$. This shows that the virtual edge is indeed longer than $E$.

In the fourth case, we cannot describe the location of the singular point as some sort of midpoint as in the earlier cases, a description which does not change when we shift. When we solve for the position of $V$ as before, and denote by $\lambda=u_{m_{a}}$ the highest weight, i.e. the coefficient of the four points $m_{a}, \ldots, m_{d}$, and by $\mu=u_{m_{e}}$ the coefficient of $m_{e}$ and $m_{f}$, then as before we get $\left(\frac{1}{3} \cdot(\lambda-\mu), 0,0\right)$ for the coordinates of $V$. The singular point is at $(0,0,0)$ which is a point of distance $\frac{\lambda-\mu}{3}$ from $V$. This distance will not change of course when we shift, however the coefficients $\lambda$ and $\mu$ are going to be changed by adding a vector in the rowspace of $A$. Since there is a unique way of writing $u$ as a sum of a vector in the weight class and a vector in the rowspace, we can in fact solve for the vector in the rowspace which we need. By our choice of coordinates for the point configuration, we can deduce that we need to add the vector of $y$-coordinates in the rowspace $\left(u_{m_{b}}-u_{m_{c}}\right)$-times and the vector of $z$-coordinates $\left(u_{m_{b}}-u_{m_{d}}\right)$-times. Then the four new coefficients of the circuit are equal, we have

$$
\begin{aligned}
\lambda & =u_{m_{a}} \\
& =u_{m_{d}}+\left(u_{m_{b}}-u_{m_{c}}\right)+2 \cdot\left(u_{m_{b}}-u_{m_{d}}\right) \\
& =u_{m_{d}}+2 \cdot\left(u_{m_{b}}-u_{m_{c}}\right)+\left(u_{m_{b}}-u_{m_{d}}\right) \\
& =u_{m_{b}}+\left(u_{m_{b}}-u_{m_{c}}\right)+\left(u_{m_{b}}-u_{m_{d}}\right) .
\end{aligned}
$$

If $M$ denotes the multiple of the $x$-vector that we add, then $M$ has to satisfy the equality

$$
\begin{aligned}
\mu & =u_{m_{f}}+\left(u_{m_{b}}-u_{m_{c}}\right) \cdot m_{f y}+\left(u_{m_{b}}-u_{m_{d}}\right) \cdot m_{f z}+3 \cdot M \\
& =u_{m_{e}}+\left(u_{m_{b}}-u_{m_{c}}\right) \cdot m_{e y}+\left(u_{m_{b}}-u_{m_{d}}\right) \cdot m_{e z}+M,
\end{aligned}
$$

where $m_{f y}$ is the second coordinate of $m_{f}$ etc., so that then the new coefficients of $m_{e}$ and $m_{f}$ are also equal. So we can solve for $M$ and then express the distance $\frac{\lambda-\mu}{3}$ 
of the singular point from $V$ as

$$
\begin{aligned}
\frac{\lambda-\mu}{3}= & \frac{u_{m_{a}}}{3}-\left(\frac{u_{m_{e}}}{2}-\frac{u_{m_{f}}}{6}\right)-\left(u_{m_{b}}-u_{m_{c}}\right) \cdot\left(\frac{m_{e y}}{2}-\frac{m_{f y}}{6}\right) \\
& -\left(u_{m_{b}}-u_{m_{d}}\right) \cdot\left(\frac{m_{e z}}{2}-\frac{m_{f z}}{6}\right) .
\end{aligned}
$$

This settles case (b.1) of Theorem 2 .

\subsection{Weight Class as in Lemma 10(b), Circuit (D) of Remark 7}

Let $F_{s-4}^{\prime}=\{a, b, c, d\}, F_{j}^{\prime}=\{e, f\}$, and assume first that $m_{e}$ and $m_{f}$ lie on different sides of the plane spanned by $m_{a}, m_{b}, m_{c}$ and $m_{d}$. Since the two points $m_{e}$ and $m_{f}$ have the biggest heights of points outside the plane, it follows that both form a pyramid with $m_{a}, m_{b}, m_{c}$ and $m_{d}$ in the subdivision. By assumption both pyramids cannot have any lattice point besides the five vertices. It follows from Lemma 3.3 of [16] that the lattice distance of both points to the plane is one. Now solve the equations given by the tropical polytope to get the positions of the two vertices corresponding to the two pyramids. Without restriction, we can assume that $m_{a}, m_{b}, m_{c}$ and $m_{d}$ lie in the $x=0$-plane, it follows that the $x$-coordinate of $m_{e}$ is -1 and the $x$-coordinate of $m_{f}$ is 1 . Since $m_{a}, m_{b}, m_{c}$ and $m_{d}$ have biggest and equal height, it follows that the edge dual to the convex hull of $m_{a}, m_{b}, m_{c}$ and $m_{d}$ satisfies the equations $y=0$ and $z=0$. If $\lambda=u_{m_{a}}$ is the biggest weight (the weight of $m_{a}, m_{b}, m_{c}$ and $m_{d}$ ), and $\mu=u_{m_{e}}$ is the weight of $m_{e}$ and $m_{f}$, it follows that the vertex dual to the pyramid with vertex $m_{e}$ is at $(\mu-\lambda, 0,0)$ and the vertex dual to the pyramid with vertex $m_{f}$ is at $(\lambda-\mu, 0,0)$. The singular point $(0,0,0)$ is thus exactly in the middle of the edge dual to the convex hull of $m_{a}, m_{b}, m_{c}$ and $m_{d}$.

Now assume $m_{e}$ and $m_{f}$ lie on the same side of the plane spanned by $m_{a}, m_{b}, m_{c}$ and $m_{d}$. It follows from Lemma 3.3 of [16] again that none of these two points can have an integral distance larger than one to the plane, or it would form a pyramid with interior lattice points. Thus both $m_{e}$ and $m_{f}$ have integral distance one, and form a "triangular roof" with $m_{a}, m_{b}, m_{c}$ and $m_{d}$. Again, then the dual subdivision does not correspond to a cone of codimension 1 of the secondary fan, and we do not consider the situation. pyramid with base $m_{a}, m_{b}, m_{c}$ and $m_{d}$. We can again solve for the position of vertex dual to this pyramid and get $(v-\lambda, 0,0)$. The singular point is on an edge ending at a vertex $V_{1}$ adjacent to five edges and nine 2-dimensional polyhedra and at a vertex $V_{2}$ with five adjacent edges and eight 2-dimensional polyhedra, and its distance to $V_{2}$ is bigger or equal to its distance to $V_{1}$. This settles case (b.2) of Theorem 2.

\subsection{Weight Class as in Lemma 10(c), Circuit (E) of Remark 7}

With the notation from Lemma 10 (c) let $F_{s-4}^{\prime}=\{a, b, c\}$ and $F_{j}^{\prime}=\{d, e, f\}$. We may assume that $m_{a}=(0,0,0), m_{b}=(0,0,1)$, and $m_{c}=(0,0,2)$. We then distinguish two cases. Either there is no plane containing the $z$-axis such that $m_{d}, m_{e}$ and $m_{f}$ are all on one side of the plane, or there is such a plane. 
Fig. 13 Lattice triangles of lattice height one

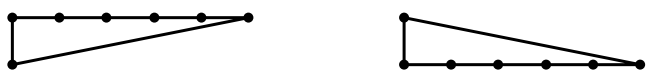

Fig. $14 \pi^{-1}(k, l)$ contains a lattice point

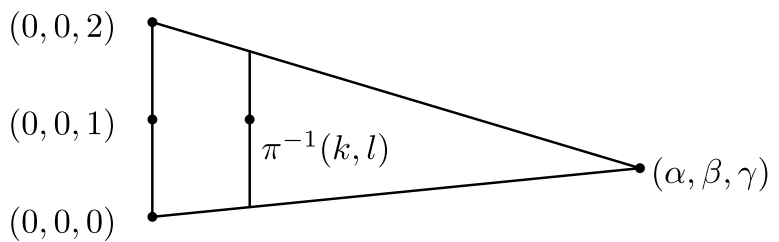

4.5.1 Assume There Is no Plane Through the $z$-Axis with $m_{d}, m_{e}$, and $m_{f}$ All on the Same Side of the Plane

In a first step we want to classify the possible polytopes spanned by $m_{a}, \ldots, m_{f}$, and then we will see how the corresponding tropical surfaces look like locally at the singular point.

Lemma 20 Let $P=\operatorname{conv}\left((0,0,0),(0,0,2), m, m^{\prime}\right)$ with $m, m^{\prime} \in \mathbb{Z}^{3}$ be a 3-dimensional lattice polytope such that

$$
P \cap \mathbb{Z}^{3}=\left\{(0,0,0),(0,0,1),(0,0,2), m, m^{\prime}\right\} .
$$

Projecting $P$ orthogonally onto the xy-plane we get a triangle $T$ which contains no interior lattice point and where the edges with vertex $(0,0)$ contain no relative interior point.

Proof We denote by $\pi: P \longrightarrow \mathbb{R}^{2}:(x, y, z) \mapsto(x, y)$ the orthogonal projection onto the $x y$-plane, so that $T=\pi(P)$.

Applying a suitable coordinate change in $\mathrm{Gl}_{3}(\mathbb{Z})$ we may assume that $m^{\prime}=$ $\left(0, \beta^{\prime}, \gamma^{\prime}\right)$ and $m=(\alpha, \beta, \gamma)$ with $\beta^{\prime}>0$. If $\beta^{\prime}>1$ then $\pi^{-1}(0,1)$ is a line segment of Euclidean length at least one and it thus contains a lattice point in contradiction to (3). Applying a coordinate change again we can assume $0 \leq \beta<\alpha$. Since $\beta^{\prime}=1$ the edge of $T$ connecting the vertex $(0,0)$ with $\left(0, \beta^{\prime}\right)$ has no relative interior point. If $\beta=0$ or $\beta=1$ the statement holds obviously, since then $T$ is a triangle of lattice height one (see Fig. 13). Note here that for $\beta=0$ necessarily $\alpha=1$ since otherwise above $\pi^{-1}(1,0)$ would contain an interior lattice point.

We may therefore assume

$$
m^{\prime}=\left(0,1, \gamma^{\prime}\right) \quad \text { and } \quad m=(\alpha, \beta, \gamma) \quad \text { with } 2 \leq \beta<\alpha .
$$

Moreover, we must have $\operatorname{gcd}(\alpha, \beta)=1$, since $\alpha=k \cdot d$ and $\beta=l \cdot d$ with $d \geq 2$ would imply that $\pi^{-1}(k, l)$ is a line segment of lattice length at least one and thus contains a lattice point in contradiction to (3), see Fig. 14. Therefore, also the edge of $T$ connecting vertex $(0,0)$ with $(\alpha, \beta)$ has no relative interior point, and if we divide $\alpha$ by $\beta$ with remainder we get

$$
\alpha=q \cdot \beta+r \quad \text { with } 1 \leq r \leq \beta-1 \text { and } q \geq 1 .
$$


The triangle $T$ can be described by inequalities as follows

$$
T=\left\{(x, y) \mid x \geq 0, y \geq \frac{\beta}{\alpha} \cdot x, y \leq \frac{\beta-1}{\alpha} \cdot x+1\right\},
$$

which ensures that

$$
(q, 1) \in T \text {. }
$$

We now want to show that

$$
\pi^{-1}(q, 1) \cap \mathbb{Z}^{3} \neq \emptyset
$$

which will be a contradiction to (3).

An easy computation shows that

$$
\pi^{-1}(q, 1)=\left\{\left(q, 1, \frac{q \cdot \gamma+r \cdot \gamma^{\prime}+z}{q \cdot \beta+r}\right) \mid 0 \leq z \leq 2 \cdot q \cdot(\beta-1)\right\},
$$

and we have to show that there is a $0 \leq z \leq 2 \cdot q \cdot(\beta-1)$ such that

$$
q \cdot \beta+r \mid\left(q \cdot \gamma+r \cdot \gamma^{\prime}\right)+z
$$

We consider first the special case $\beta=2$. Then necessarily $r=1$ and there is of course a $0 \leq z \leq 2 \cdot q$ such that $q \cdot \beta+r=2 \cdot q+1$ divides $\left(q \cdot \gamma+\gamma^{\prime}\right)+z$.

Next we consider the special case $(q, r)=(1, \beta-1)$, and we have to check if $q \cdot \beta+r=2 \cdot \beta-1$ divides $\left(\gamma+(\beta-1) \cdot \gamma^{\prime}\right)+z$ for some $0 \leq z \leq 2 \cdot \beta-2$, which is obviously the case.

For the general case we may now assume that $\beta \geq 3$ and $(q, r) \neq(1, \beta-1)$. Taking (4) and (5) into account it follows that

$$
\beta \geq 2+\frac{r}{q}
$$

or equivalently

$$
2 \cdot q \cdot(\beta-1) \geq q \cdot \beta+r .
$$

But then, there is definitely a $0 \leq z \leq 2 \cdot q \cdot(\beta-1)$ such that (6) is satisfied.

So the case $2 \leq \beta<\alpha$ cannot occur, and this finishes the proof.

Proposition 21 Let $P$ be a lattice polytope which is the convex hull of a circuit of type $(E)$ and three additional lattice points $m, m^{\prime}$ and $m^{\prime \prime}$ such that any two of these together with the circuit span $\mathbb{R}^{3}, P$ contains only the given six lattice points, and there is no plane through the z-axis such that $m, m^{\prime}$ and $m^{\prime \prime}$ are all on the same side of the plane, see Fig. 15.

Then the circuit is given up to IUA-equivalence by $(0,0,0),(0,0,1)$, and $(0,0,2)$, and the lattice points $m, m^{\prime}$, and $m^{\prime \prime}$ satisfy the conditions in exactly one of the following cases: 
Fig. 15 A lattice polytope $P$ as in Proposition 21 with subdivision
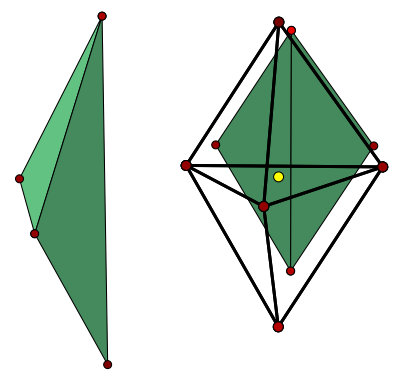

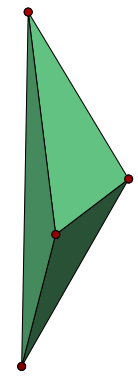

Fig. $16 \pi(P)=T \cup T^{\prime} \cup T^{\prime \prime}$ decomposes as a union of three triangles
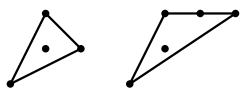

$T_{1}$

$T_{2}$

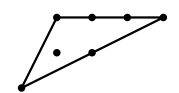

$T_{3}$

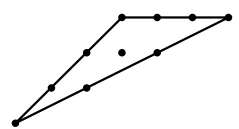

$T_{4}$
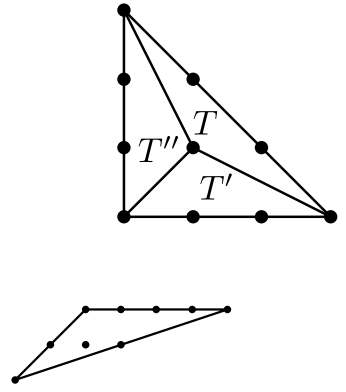

$T_{5}$

Fig. 17 The five lattice triangles with one interior lattice point

(a) $m=(0,1, \gamma), m^{\prime}=\left(1,0, \gamma^{\prime}\right)$, and $m^{\prime \prime}=\left(-1,-1, \gamma^{\prime \prime}\right)$ with $\gamma, \gamma^{\prime}, \gamma^{\prime \prime} \in \mathbb{Z}$ arbitrary.

(b) $m=(0,1, \gamma), m^{\prime}=\left(2,1, \gamma^{\prime}\right)$, and $m^{\prime \prime}=\left(-1,-1, \gamma^{\prime \prime}\right)$ with $\gamma, \gamma^{\prime}, \gamma^{\prime \prime} \in \mathbb{Z}$ such that $\gamma \not \equiv \gamma^{\prime}(\bmod 2)$.

(c) $m=(0,1, \gamma), m^{\prime}=\left(3,1, \gamma^{\prime}\right)$, and $m^{\prime \prime}=\left(-1,-1, \gamma^{\prime \prime}\right)$ with $\gamma, \gamma^{\prime}, \gamma^{\prime \prime} \in \mathbb{Z}$ such that $\gamma \not \equiv \gamma^{\prime}(\bmod 3)$ and $\gamma^{\prime} \not \equiv \gamma^{\prime \prime}(\bmod 2)$.

(d) $m=(0,1, \gamma), m^{\prime}=\left(3,1, \gamma^{\prime}\right)$, and $m^{\prime \prime}=\left(-3,-2, \gamma^{\prime \prime}\right)$ with $\gamma, \gamma^{\prime}, \gamma^{\prime \prime} \in \mathbb{Z}$ such that $\gamma \not \equiv \gamma^{\prime} \not \equiv \gamma^{\prime \prime} \not \equiv \gamma(\bmod 3)$.

Proof It is clear that the circuit (E) is IUA-equivalent to $(0,0,0),(0,0,1)$, and $(0,0,2)$. If we denote by $\pi: P \longrightarrow \mathbb{R}^{2}:(x, y, z) \mapsto(x, y)$ the projection onto the $x y$-plane then $\pi(P)$ is a triangle which decomposes into three triangles $\pi(P)=$ $T \cup T^{\prime} \cup T^{\prime \prime}$ as in Lemma 20, see Fig. 16. Lemma 20 therefore implies that $(0,0)$ is the only interior lattice point of $\pi(P)$. Lattice polygons with exactly one interior lattice point have been classified up to IUA-equivalence, see e.g. [14] or [13], and among them are exactly five triangles as shown in Fig. 17, where the interior lattice point is $(0,0)$. Applying a $\mathbb{Z}$-linear coordinate change we may therefore assume that $\pi(P)$ is one of these five triangles. In each of the cases it remains to check whether there exist polytopes $P$ that project to the triangle and what restrictions this poses on the third component of the lattice points $m, m^{\prime}$, and $m^{\prime \prime}$. Actually, the only obstruction is that above the relative interior lattice points on the edges of the triangles 
Fig. 18 The triangle in the tropical surface dual to the circuit

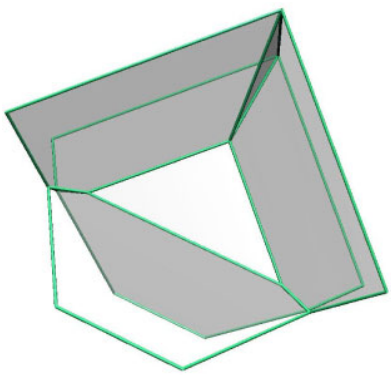

there should be no lattice point in $P$. If such an edge has $k$ relative interior lattice points and the $z$-coordinates of the vertices of the edge differ by $l$, then some of the relative interior lattice points lifts to a lattice point if and only if $k+1$ and $l$ are not coprime. Therefore, $T_{1}, \ldots, T_{4}$ lead to the four cases mentioned in the statement of the proposition. For $T_{5}$ we would need points $m=(0,1, \gamma), m^{\prime}=\left(4,1, \gamma^{\prime}\right)$, and $m^{\prime \prime}=\left(-2,-1, \gamma^{\prime \prime}\right)$ such that each of the differences $\gamma-\gamma^{\prime}, \gamma-\gamma^{\prime \prime}$ and $\gamma^{\prime}-\gamma^{\prime \prime}$ is coprime to two. That is obviously not possible, so that $T_{5}$ cannot be the projection of any $P$.

In order to understand how the tropicalization of the singular point locally looks like in the case we are considering, assume first that the subdivision contains a polytope as considered in Proposition 21, and it is subdivided into the three polytopes $\Delta_{1}=\operatorname{conv}\left(m_{a}, m_{c}, m_{d}, m_{e}\right), \Delta_{2}=\operatorname{conv}\left(m_{a}, m_{c}, m_{d}, m_{f}\right)$ and $\Delta_{3}=$ $\operatorname{conv}\left(m_{a}, m_{c}, m_{e}, m_{f}\right)$, see Fig. 15 . The circuit $\left\{m_{a}, m_{b}, m_{c}\right\}$ is then dual to a triangle in the tropical surface whose vertices are dual to $\Delta_{1}, \Delta_{2}$, and $\Delta_{3}$, see Fig. 18. We assume as before that $m_{a}=(0,0,0), m_{b}=(0,0,1)$ and $m_{c}=(0,0,2)$. Recall that we can project $P$ to the $(x, y)$-plane and obtain three triangles $T, T^{\prime}$ and $T^{\prime \prime}$ as in Fig. 16. The midpoint is $(0,0)$. Denote the coordinates of the three vertices by $\left(r_{1}, s_{1}\right),\left(r_{2}, s_{2}\right)$ and $\left(r_{3}, s_{3}\right)$. Let us use the tropical polynomial to solve for the coordinates $(x, y, z)$ of the three vertices dual to $\Delta_{1}, \Delta_{2}$, and $\Delta_{3}$. By assumption the heights associated to the lattice points satisfy $u_{m_{a}}=u_{m_{b}}=u_{m_{c}}$ and $u_{m_{d}}=u_{m_{e}}=u_{m_{f}}$, and we set $u=u_{m_{a}}-u_{m_{d}}$. For any $i=1,2,3$, the equation $u+z=u$ has to be satisfied, so any of the three vertices has $z$-coordinate 0 . In fact, the whole triangle dual to the circuit satisfies $z=0$. So we only have to solve for the $(x, y)$-coordinates of the vertices. For any choice of $(i, j)=(1,2),(2,3)$ or $(3,1)$, the vertex dual to the polytope which projects to the triangle spanned by $(0,0),\left(r_{i}, s_{i}\right)$ and $\left(r_{j}, s_{j}\right)$ has to satisfy the equations $u=r_{i} x+s_{i} y$ and $u=r_{j} x+s_{j} y$, which are solved by $(x, y)=\frac{1}{r_{i} s_{j}-s_{i} r_{j}} \cdot\left(s_{j} u-s_{i} u, r_{i} u-r_{j} u\right)$. Now assign to each of the vertices the area of the projection of the dual polytope, i.e. $\left(r_{i} s_{j}-s_{i} r_{j}\right)$, as weight. Then it follows that the weighted sum of the three vertices is $(0,0,0)$, i.e. the singular point. Thus, the singular point tropicalizes precisely to the weighted barycenter of the triangle dual to the circuit. Figure 19 depicts this situation for the case that the projection is the triangle $T_{3}$ of Fig. 17.

If the subdivision locally around the circuit contains further lattice points, the local picture may look more complicated. However, the circuit $\left\{m_{a}, m_{b}, m_{c}\right\}$ is still dual to a polygon $Q$ in the $\{z=0\}$-plane. Moreover, in the subdivision there will still be 

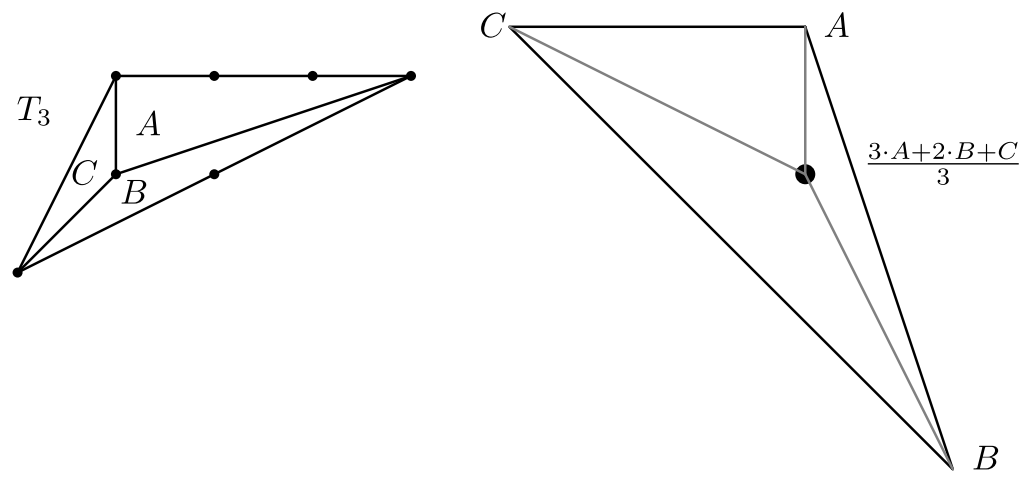

Fig. $19 T_{3}=\pi(\Delta)$ and the dual triangle in the tropical surface showing $(0,0,0)$ as the weighted barycenter $\frac{3 \cdot A+2 \cdot B+C}{3}$

Fig. 20 The origin as a generalized weighted barycenter

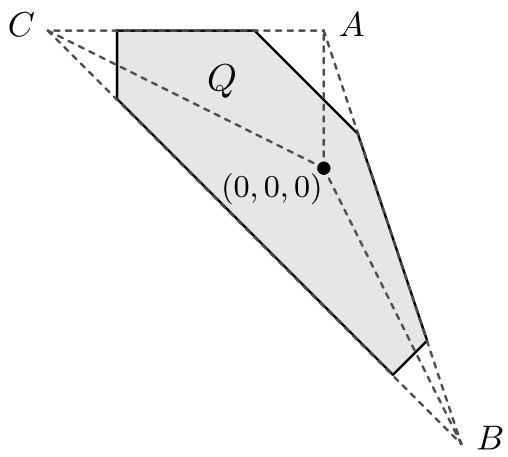

polytopes which contain $\operatorname{conv}\left(m_{a}, m_{c}, m_{d}\right)$ respectively $\operatorname{conv}\left(m_{a}, m_{c}, m_{e}\right)$ respectively $\operatorname{conv}\left(m_{a}, m_{c}, m_{f}\right)$ as a facet. Therefore, the polygon $Q$ will have three edges dual to these facets. If one computes the intersection points of the lines through these edges, one gets three points $A, B$, and $C$ which would be dual to the polytopes $\Delta_{i}$. This extension of the cell forms a virtual triangular cell, and the tropicalization of the singular point is still the weighted sum of the three points $A, B$ and $C$, see Fig. 20.

Example 22 A concrete example for this behavior is the singular point $H=$ $(-1,-1,0)$ on the tropical surface in Example 3. We have seen in Remark 17 which weight class corresponds to the point $H=(-1,-1,0)$. We have

$$
\begin{array}{lll}
m_{a}=(0,0,0), & m_{b}=(0,0,1), & m_{c}=(0,0,2), \\
m_{d}=(-1,-1,0), & m_{e}=(0,1,0), & m_{f}=(1,0,0),
\end{array}
$$

and one further point $m_{g}=(1,2,1)$. The circuit $m_{a}, m_{b}, m_{c}$ corresponds then to quadrangle $A B C D$ (see Fig. 21, where the vertices $C=(5,-13,0)$ and $D=(-13,5,0)$ correspond to the polytopes $\Delta_{C}=\operatorname{conv}\left\{m_{a}, m_{c}, m_{d}, m_{e}\right\}$ respectively $\Delta_{D}=\operatorname{conv}\left\{m_{a}, m_{c}, m_{d}, m_{f}\right\}$ in the subdivision. The polytope $\Delta_{E}=$ $\operatorname{conv}\left\{m_{a}, m_{c}, m_{e}, m_{f}\right\}$, however, is not part of the subdivision due to the presence 
Fig. 21 The singular point as barycenter

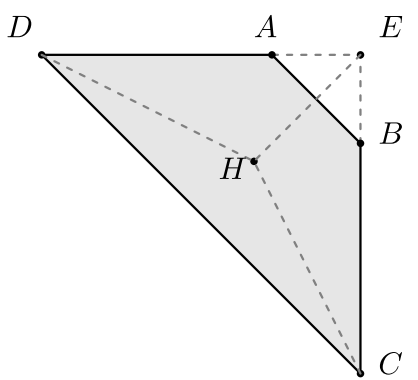

of $m_{g}$ with an appropriate height. However, $\Delta_{E}$ defines a virtual point $E=(5,5,0)$, which is the intersection of the two lines determined by the facets $\operatorname{conv}\left\{m_{a}, m_{c}, m_{e}\right\}$ and $\operatorname{conv}\left\{m_{a}, m_{c}, m_{f}\right\}$ of $\Delta_{C}$ respectively $\Delta_{D}$, and

$$
H=\frac{1}{3} \cdot(C+D+E)
$$

is the barycenter of this virtual triangle in the tropical surface.

4.5.2 Assume There Is a Plane Through the $z$-Axis with $m_{d}, m_{e}$, and $m_{f}$ All on the Same Side of the Plane

Again we first want to classify the possible polytopes spanned by $m_{a}, \ldots, m_{f}$, and then we will see how the corresponding tropical surfaces look like locally at the singular point.

Proposition 23 Let $P$ be a lattice polytope which is the convex hull of a circuit of type $(E)$ and three additional lattice points $m, m^{\prime}$, and $m^{\prime \prime}$ such that any two of these together with the circuit span $\mathbb{R}^{3}, P$ contains only the given six lattice points, and there is a plane through the z-axis such that $m, m^{\prime}$ and $m^{\prime \prime}$ are all on the same side of the plane, see Fig. 26.

Then the circuit is given up to IUA-equivalence by $(0,0,0),(0,0,1)$, and $(0,0,2)$, and the lattice points $m, m^{\prime}$, and $m^{\prime \prime}$ (up to reordering) satisfy the conditions in exactly one of the following cases:

(a) $m=(-1,0, \gamma), m^{\prime}=\left(0,1, \gamma^{\prime}\right)$, and $m^{\prime \prime}=\left(\alpha^{\prime \prime}, 1, \gamma^{\prime \prime}\right)$ with $\alpha^{\prime \prime} \geq 1, \gamma \in \mathbb{Z}$ arbitrary and $\operatorname{gcd}\left(\gamma^{\prime \prime}-\gamma^{\prime}, \alpha^{\prime \prime}\right)=1$.

(b) $m=(\alpha, 1, \gamma), m^{\prime}=(\alpha+l, 1, \gamma+k)$, and $m^{\prime \prime}=(\alpha+2 \cdot l, 1, \gamma+2 \cdot k)$ with $\alpha, \gamma \in \mathbb{Z}$ arbitrary and $\operatorname{gcd}(l, k)=1$.

(c) $m=(\alpha, 1, \gamma), m^{\prime}=\left(\alpha^{\prime}, 1, \gamma^{\prime}\right)$, and $m^{\prime \prime}=\left(\alpha^{\prime \prime}, 1, \gamma^{\prime \prime}\right)$ with

$$
\operatorname{det}\left(\begin{array}{ll}
\alpha^{\prime}-\alpha & \alpha^{\prime \prime}-\alpha \\
\gamma^{\prime}-\gamma & \gamma^{\prime \prime}-\gamma
\end{array}\right)= \pm 1
$$

Proof Up to IUA-equivalence we may assume that the circuit is $(0,0,0),(0,0,1)$, and $(0,0,2)$. Projecting $\Delta$ to the $x y$-plane the points $\pi(m), \pi\left(m^{\prime}\right)$, and $\pi\left(m^{\prime \prime}\right)$ lie in 

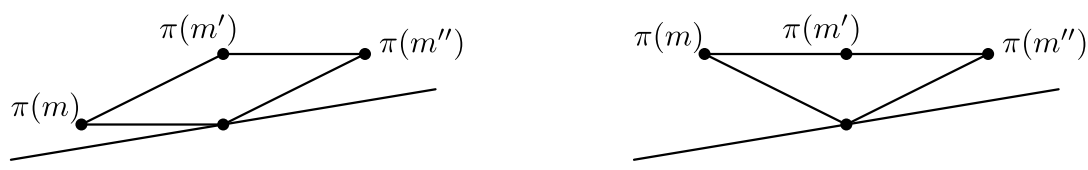

Fig. $22 \pi(\Delta)$ with the separating hyperplane

Fig. 23 The normal form of $\pi(\Delta)$ when it is a triangle

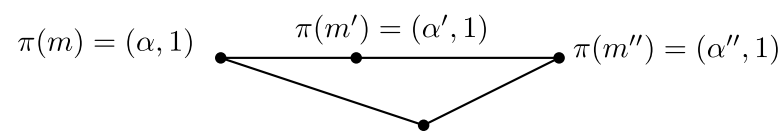

one half plane. Due to the assumptions on $\Delta$ no two of these points lie on the same line through the origin, and ordering these lines by their angle clockwise we may assume up to reordering that the points $\pi(m), \pi\left(m^{\prime}\right)$, and $\pi\left(m^{\prime \prime}\right)$ come in this order, see Fig. 22 for possible configurations.

We should note here first that in $\pi(\Delta)$ the point $\pi\left(\mathrm{m}^{\prime}\right)$ cannot be an interior point of $\operatorname{conv}\left((0,0), \pi(m), \pi\left(m^{\prime \prime}\right)\right)$, since otherwise $\operatorname{conv}\left((0,0,0),(0,0,2), m, m^{\prime \prime}\right)$ will be in the subdivision of $\Delta$ which therefore satisfies the assumptions on Lemma 20, but $\pi\left(m^{\prime}\right)$ would violate these assumptions. It is then natural to distinguish the two cases that either $\pi\left(m^{\prime}\right)$ is on the line segment connecting $\pi(m)$ and $\pi\left(m^{\prime \prime}\right)$, i.e. $\pi(\Delta)$ is a triangle as shown on the right hand side of Fig. 22, or $\pi(\Delta)$ is a quadrangle as shown on the left hand side of Fig. 22. In any case, applying Lemma 20 to the convex hull of the circuit and two of the further lattice points $m, m^{\prime}$, and $m^{\prime \prime}$, we see that each of the points $\pi(m), \pi\left(m^{\prime}\right)$, and $\pi\left(m^{\prime \prime}\right)$ has lattice distance one from the origin.

Let us first consider the case that $\pi(\Delta)$ is a triangle. Up to IUA-equivalence we may assume that the line through $\pi(m), \pi\left(m^{\prime}\right)$, and $\pi\left(m^{\prime \prime}\right)$ is parallel to the $x$-axis, i.e. $\pi(m)=(\alpha, \beta), \pi\left(m^{\prime}\right)=\left(\alpha^{\prime}, \beta\right)$, and $\pi\left(m^{\prime \prime}\right)=\left(\alpha^{\prime \prime}, \beta\right)$ with $\alpha<\alpha^{\prime}<\alpha^{\prime \prime}$. By Lemma 20 the triangle $\operatorname{conv}\left((0,0), \pi(m), \pi\left(m^{\prime \prime}\right)\right)$ has no interior lattice point and the number of lattice points on the boundary is $\alpha^{\prime \prime}-\alpha+2$, so that Pick's Formula implies $\beta=1$, see Fig. 23 .

This case now subdivides into two subcases, namely, that the points $m, m^{\prime}$, and $m^{\prime \prime}$ lie on a line, respectively that they form a triangle. If the three points lie on a line, then $m^{\prime}$ must be the midpoint of the line segment from $m$ to $m^{\prime \prime}$ and the line segment contains no further lattice point. Thus, $\operatorname{gcd}\left(\alpha^{\prime \prime}-\alpha, \gamma^{\prime \prime}-\gamma\right)=2$ is the only obstruction that has to be satisfied, and we are thus in Case (b) of the proposition with $l=\frac{\alpha^{\prime \prime}-\alpha}{2}$ and $k=\frac{\gamma^{\prime \prime}-\gamma}{2}$. If the three points $m, m^{\prime}$, and $m^{\prime \prime}$ form a triangle, then the only obstruction to the condition that $\Delta$ contains no further lattice points is that this triangle should have lattice area one. This is precisely the condition of Case (c) in the proposition.

It remains to consider the case that $\pi(\Delta)$ is a quadrangle. As in the proof of Lemma 20, up to IUA-equivalence, $m^{\prime}=\left(0,1, \gamma^{\prime}\right)$ and $m^{\prime \prime}=\left(\alpha^{\prime \prime}, \beta^{\prime \prime}, \gamma^{\prime \prime}\right)$ with $0 \leq \beta^{\prime \prime}<\alpha^{\prime \prime}$. Moreover, since the triangle $T=\operatorname{conv}\left((0,0), \pi\left(m^{\prime}\right), \pi\left(m^{\prime \prime}\right)\right)$ contains no interior lattice point due to Lemma 20 Pick's Formula implies that $\beta^{\prime \prime} \in\{0,1\}$, and if $\beta^{\prime \prime}=1$ then necessarily $\alpha^{\prime \prime}=1$, since the lattice distance from $\pi\left(m^{\prime \prime}\right)$ to the origin is one. See Fig. 24. 

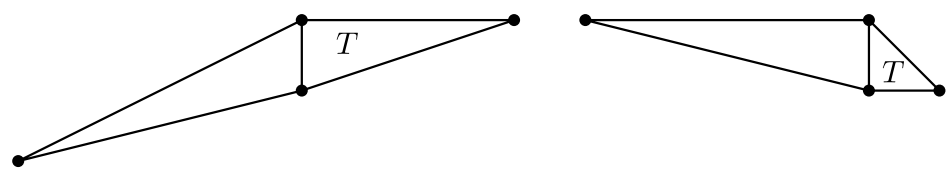

Fig. 24 Possible configurations for the triangle $T=\operatorname{conv}\left((0,0), \pi\left(m^{\prime}\right), \pi\left(m^{\prime \prime}\right)\right)$
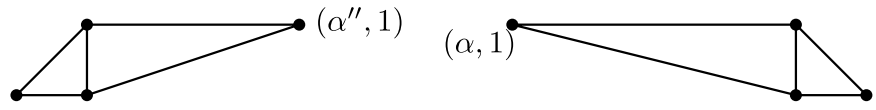

Fig. 25 The normal forms of $\pi(\Delta)$ when it is a quadrangle
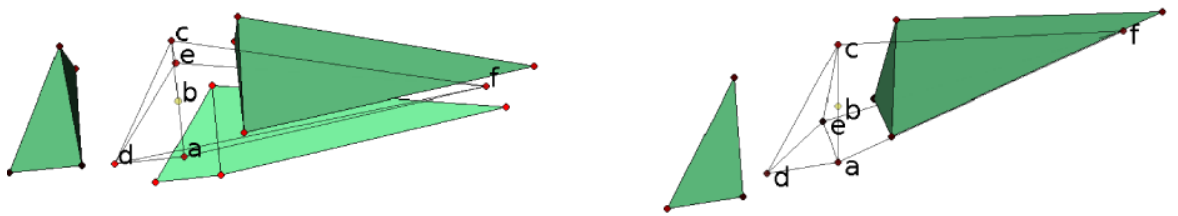

Fig. 26 Possible subdivisions of $\Delta$

Let us now consider the case $\beta^{\prime \prime}=1$ in more detail. The point $\pi(m)=(\alpha, \beta)$ has to lie below the line $\{y=1\}$ and above the line $\{\alpha \cdot y=x\}$. Thus $0 \geq \beta>\alpha$, and applying Pick's Formula once again we find $\beta=0$, and then necessarily $\alpha=-1$. Analogously, we get in the case $\beta^{\prime \prime}=0$ that $\beta^{\prime \prime}=1$ and $\alpha \geq 1$. That is, $\pi(\Delta)$ is one of the quadrangles shown in Fig. 25.

Obviously, reflecting at the plane $\{x=0\}$ and exchanging $m$ and $m^{\prime \prime}$ the two possible configuration types are equivalent, so that we may assume that $\beta=1$. We thus have $m=(-1,0, \gamma), m^{\prime}=\left(0,1, \gamma^{\prime}\right)$, and $m^{\prime \prime}=\left(\alpha^{\prime \prime}, 1, \gamma^{\prime \prime}\right)$. Only above the line segment joining $\pi\left(\mathrm{m}^{\prime}\right)$ and $\pi\left(\mathrm{m}^{\prime \prime}\right)$ there could be an additional lattice point in $\Delta$ if the coordinates $\gamma^{\prime}$ and $\gamma^{\prime \prime}$ are chosen inappropriately, and the condition to avoid this is $\operatorname{gcd}\left(\gamma^{\prime \prime}-\gamma^{\prime}, \alpha^{\prime \prime}\right)=1$. We are thus in Case (a) of the proposition, and this finishes the proof.

We now have to see how the tropical surface looks locally at the tropicalization of the singular point, i.e. locally at $(0,0,0)$. As in Sect. 4.5.1 we want to restrict first to the case where the Newton polytope $\Delta$ is just the convex hull of $m_{a}, \ldots, m_{f}$, and in the notation of Proposition 23 we may assume that $m_{d}=m, m_{e}=m^{\prime}$, and $m_{f}=m^{\prime \prime}$. Moreover, we will consider the Case (a) in Proposition 23 first. In the subdivision of $\Delta$ there will be exactly two polytopes which contain the circuit $m_{a}, m_{b}$, and $m_{c}$, namely $\Delta_{A}=\operatorname{conv}\left(m_{a}, m_{b}, m_{c}, m_{d}, m_{e}\right)$ and $\Delta_{B}=\operatorname{conv}\left(m_{a}, m_{b}, m_{c}, m_{e}, m_{f}\right)$, see Fig. 26. The subdivision may contain a third polytope $\operatorname{conv}\left(m_{a}, m_{d}, m_{e}, m_{f}\right)$, respectively, $\operatorname{conv}\left(m_{c}, m_{d}, m_{e}, m_{f}\right)$ which does not contain the circuit, and which consequently will not matter for the singular point.

The tropicalization of the singular point will then be contained in the plane segment dual to the circuit. This segment will be unbounded, but it has two vertices 
Fig. 27 The singular point at the virtual barycenter.

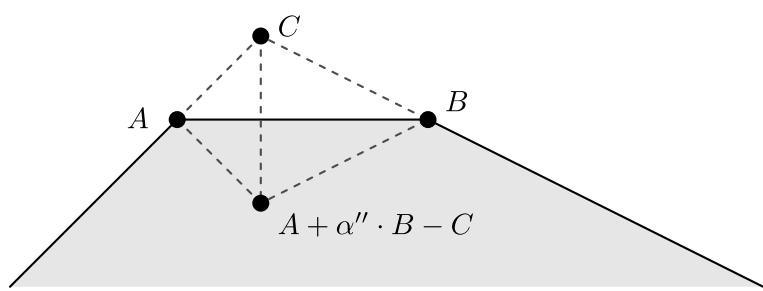

$A$ and $B$ which are dual the polytopes $\Delta_{A}$ and $\Delta_{B}$. Moreover, if we consider the lines through the line segments which are dual to $\operatorname{conv}\left(m_{a}, m_{b}, m_{c}, m_{d}\right)$ and $\operatorname{conv}\left(m_{a}, m_{b}, m_{c}, m_{f}\right)$ respectively, then these will intersect in a point $C$ which is dual to the polytope $\operatorname{conv}\left(m_{a}, m_{b}, m_{c}, m_{d}, m_{f}\right)$ which is not part of the subdivision. Anyway, if we assign to the points $A, B$, and $C$ as weights the lattice area of the corresponding triangle in $\pi(\Delta)$, e.g. $B$ gets as weight the lattice area $\alpha^{\prime \prime}$ of $\operatorname{conv}\left((0,0),(0,1),\left(\alpha^{\prime \prime}, 1\right)\right)$, and if we moreover consider the weight of $C$ negatively, since $C$ lies outside the plane segment, then the tropicalization of the singular point is the weighted sum of $A, B$, and $C$. In the normal form a simple computation gives $A=(-u, u, 0), B=(0, u, 0)$ and $C=\left(-u,\left(1+\alpha^{\prime \prime}\right) \cdot u, 0\right)$, and $\frac{A+\alpha^{\prime \prime} \cdot B-C}{3}=(0,0,0)$. We could thus interpret the tropicalization of the singular point as a virtual weighted barycenter of the virtual triangle $A B C$, see Fig. 27.

In our classification we need not consider the Case (b) in Proposition 23, since there the weight class $C$ in $\operatorname{Trop}(\operatorname{Ker}(A))$ corresponding to this situation is defective because $\operatorname{span}(C)$ intersects the lineality space in the vector corresponding to the $y$ coordinates of the point configuration.

The Case (c) in Proposition 23 differs from Case (a) by the fact that the points $A$, $B$, and $C$ all coincide, and that the plane segment corresponding to the circuit has only one vertex. However, it remains true that the tropicalization of the singular point is the weighted sum of $A, B$, and $C$.

Finally, if the Newton polytope contains further points the situation becomes more complicated. The polytopes $\Delta_{A}$ and $\Delta_{B}$ might be subdivided further, and consequently the vertices $A$ and $B$ might be cut off, similar to the situation described in Fig. 20. As in Sect. 4.5.1 we can still identify the virtual points $A, B$, and $C$ and their weighted sum is the tropicalization of the singular point.

Example 24 A concrete example for this behavior is the singular point $G=(0,0,0)$ on the tropical surface in Example 3. Here

$$
\begin{array}{lll}
m_{a}=(0,0,0), & m_{b}=(0,0,1), & m_{c}=(0,0,2), \\
m_{d}=(1,2,1), & m_{e}=(0,1,0), & m_{f}=(1,0,0),
\end{array}
$$

and one further point $m_{g}=(-1,-1,0)$. Note that the points $m_{d}, m_{e}, m_{f}$ are all on the same side of the plane $x+y=0$ through the circuit. The circuit $m_{a}, m_{b}, m_{c}$ corresponds then to quadrangle $A B C D$ (see Fig. 28, where the vertices $A=(0,5,0)$ and $B=(5,0,0)$ correspond to the polytopes $\Delta_{A}=\operatorname{conv}\left\{m_{a}, m_{c}, m_{d}, m_{e}\right\}$ respectively $\Delta_{B}=\operatorname{conv}\left\{m_{a}, m_{c}, m_{d}, m_{f}\right\}$ in the subdivision. The polytope $\Delta_{E}=$ $\operatorname{conv}\left\{m_{a}, m_{c}, m_{e}, m_{f}\right\}$, however, is not part of the subdivision and defines only a 
Fig. 28 The singular point as barycenter.

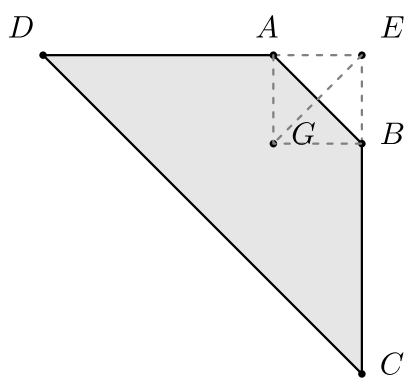

virtual point $E=(5,5,0)$, which is the intersection of the two lines determined by the facets $\operatorname{conv}\left\{m_{a}, m_{c}, m_{e}\right\}$ and $\operatorname{conv}\left\{m_{a}, m_{c}, m_{f}\right\}$ of $\Delta_{A}$ respectively $\Delta_{B}$. In this situation and

$$
G=\frac{1}{3} \cdot(C+D-E)
$$

is the virtual weighted barycenter of this virtual triangle in the tropical surface. Note here that the virtual vertex $E$ comes with a negative weight since it lies outside the plane segment dual to the circuit even if we only consider the points $m_{a}, \ldots, m_{f}$. Note also that the plane segment dual to the circuit is bounded due to the presence of the additional point $m_{g}$.

\subsection{Weight Class as in Lemma 10(d), Circuit (E) of Remark 7}

Let $F_{s-4}^{\prime}=\{a, b, c\}, F_{j}^{\prime}=\{d, e\}$ and $F_{i}^{\prime}=\{f, g\}$. We assume without restriction that $m_{a}=(0,0,0), m_{b}=(0,0,1)$ and $m_{c}=(0,0,2)$. Dual to this circuit is then as before a 2-dimensional polyhedron satisfying $z=0$. We know that in this situation, the points $m_{d}$ and $m_{e}$ lie in a plane with the line $\{x=y=0\}$, we can assume that this plane satisfies $y=0$. Let us first assume that $m_{d}$ and $m_{e}$ lie on different sides of the line, i.e. we assume that $m_{d}$ has positive $x$-coordinate and $m_{e}$ has negative $x$-coordinate. Then the triangle with vertices $m_{a}, m_{c}$ and $m_{d}$ (resp. $m_{e}$ ) will be a face of a polytope in the subdivision. If $m_{d}$ or $m_{e}$ had integral distance bigger one from the circuit, this face would contain extra lattice points, contradicting our assumption that the surface is of maximal-dimensional geometric type. It follows that $m_{d}$ has $x$-coordinate 1 and $m_{e}$ has $x$-coordinate -1 . Also, the triangle spanned by $m_{a}, m_{c}$ and $m_{f}$ (resp. $m_{g}$ ) are faces of the subdivision and thus $m_{f}$ and $m_{g}$ must have integral distance one to the plane $\{y=0\}$. Let us first assume $m_{f}$ has $y$-coordinate 1 and $m_{g}$ has $y$-coordinate -1 . Assume first that the subdivision locally contains only the polytopes $\operatorname{conv}\left(m_{a}, m_{c}, m_{d}, m_{f}\right), \operatorname{conv}\left(m_{a}, m_{c}, m_{e}, m_{f}\right)$, $\operatorname{conv}\left(m_{a}, m_{c}, m_{d}, m_{g}\right)$ and $\operatorname{conv}\left(m_{a}, m_{c}, m_{e}, m_{g}\right)$. Then corresponding to this part of the subdivision we have a quadrangle on the surface. Let us solve for the $(x, y)$ coordinates of the four vertices. Assume $m_{d}=(1,0, \gamma), m_{e}=\left(-1,0, \gamma^{\prime}\right), m_{f}=$ $\left(\alpha, 1, \gamma^{\prime \prime}\right)$ and $m_{g}=\left(\alpha^{\prime},-1, \gamma^{\prime \prime \prime}\right)$. Let us denote by $u=u_{m_{a}}-u_{m_{d}}$ the difference of the weights of $m_{a}$ and $m_{d}$ and by $w=u_{m_{d}}-u_{m_{f}}$ the difference of the weights of $m_{d}$ and $m_{f}$. Then the coordinates of the four vertices are $A=(u, w+(1-\alpha) u)$, 
Fig. 29 The trapeze with the singular point as its midpoint, and the more general situation
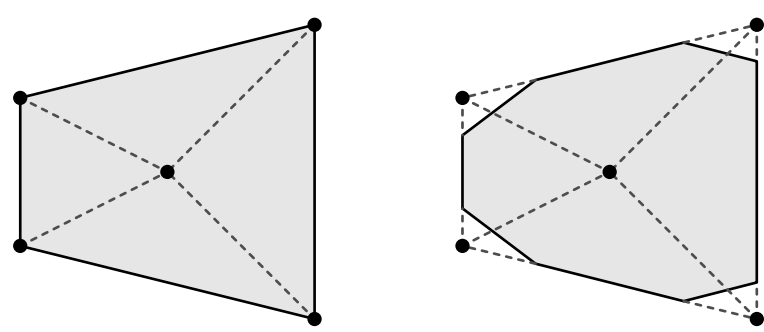

$B=(-u, w+(1+\alpha) u), C=\left(u,-w+\left(\alpha^{\prime}-1\right) u\right)$ and $D=\left(-u,-w-\left(1+\alpha^{\prime}\right) u\right)$. That is, the quadrangle is a trapeze with the singular point $(0,0,0)=\frac{A+B+C+D}{4}$ as its midpoint, as depicted in Fig. 29 on the left.

If the subdivision contains more polytopes than just these four locally around the circuit, then we get a polygon with more sides. The four edges of the trapeze are still present, and the singular point is still the midpoint. We can thus extend the cell to a virtual trapeze cell. This more general situation is depicted in Fig. 29 on the right.

If $m_{d}$ and $m_{e}$ are on the same side of the circuit in the plane $\{y=0\}$, then they must both be of integral distance one, and they form a quadrangle with the circuit which is a face of the subdivision. Thus the dual subdivision does not correspond to a cone of the secondary fan of codimension 1 , and we do not consider the situation. Analogously, if $m_{f}$ and $m_{g}$ are on the same side of the plane $\{y=0\}$, they must both have integral distance one to $\{y=0\}$. However, since the edge connecting $m_{f}$ and $m_{g}$ and the circuit do not need to lie in a plane, it may be that only one of the points $m_{f}$ or $m_{g}$ forms a facet of the subdivision with the circuit. In this case, the dual subdivision corresponds to a cone of codimension 1 . However, since the span of the corresponding weight class intersects the rowspace of $A$ non-trivially (both contain the vector of $x$-coordinates of the points $m \in \mathcal{A}$ ), this weight class is defective and we do not consider the situation.

Acknowledgements We would like to thank Christian Haase for useful discussions. The images were obtained with the aid of Polymake [7], Javaview [12], jReality [9], tropicalinsect [2], xfig and texdraw. The authors were supported by the Hermann-Minkowski Minerva Center for Geometry at the Tel Aviv University, and by the DFG-grant MA 4797/3-1 as part of the priority program SPP 1489. The third author was also supported by the Israeli Science Foundation grant no. 448/09. We would like to thank an anonymous referee for valuable comments on a first draft of this paper.

\section{References}

1. Ardila, F., Klivans, C.: The Bergman complex of a matroid and phylogenetic trees. J. Combin. Theory Ser. B 96, 38-49 (2006)

2. Bindel, N., Kopp, H.: TROPICALINSECT 05.9. Technical report, TU Kaiserslautern (2011)

3. Dickenstein, A., Feichtner, E.M., Sturmfels, B.: Tropical discriminants. J. Amer. Math. Soc. 20, 11111133 (2007). arXiv:math.AG/0510126

4. Dickenstein, A., Tabera, L.F.: Singular tropical hypersurfaces. Disc. Comp. Geom. 47(2), 430-453 (2012). arXiv: 1012.5812

5. Einsiedler, M., Kapranov, M., Lind, D.: Non-archimedean amoebas and tropical varieties. J. Reine Angew. Math. 601, 139-157 (2006)

6. Feichtner, E.M., Sturmfels, B.: Matroid polytopes, nested sets and Bergman fans. Portugaliae Mathematica 62, 437-468 (2005). arXiv:math.CO/0411260 
7. Gawrilow, E., Joswig, M.: POLYMAKE 2.9.10. Technical report, TU Berlin and TU Darmstadt (2011) http://www.math.tu-berlin.de/polymake

8. Gelfand, I.M., Kapranov, M.M., Zelevinsky, A.V.: Discriminants, Resultants, and Multidimensional Determinants. Birkhäuser, Boston (1994)

9. Gunn, C., Hoffmann, T., Schmies, M., Weissmann, S.: JREALITY. Technical report, TU Berlin (2007)

10. Markwig, H., Markwig, T., Shustin, E.: Tropical curves with a singularity in a fixed point. Man. Math. 137(3-4), 383-418 (2011). arXiv:0909.1827

11. Mikhalkin, G.: Enumerative tropical geometry in $\mathbb{R}^{2}$. J. Amer. Math. Soc. 18, 313-377 (2005). arXiv:math.AG/0312530

12. Polthier, K.: JAVAVIEW v3.95. Technical report (2006). http://www.javaview.de

13. Poonen, B., Rodriguez-Villegas, F.: Lattice polygons and the number 12. Amer. Math. Monthly 107(3), 238-250 (2000)

14. Rabinowitz, S.: A census of convex lattice polygons with at most one interior lattice point. Ars Combin. 28, 83-96 (1989)

15. Reznick, B.: Clean lattice tetrahedra (2006). Preprint. arXiv:math/0606227

16. Scarf, H.E.: Integral polyhedra in three space. Math. Oper. Res 10(3), 403-438 (1985)

17. Sturmfels, B.: Solving Systems of Linear Equations. CBMS Regional Conference Series in Mathematics, vol. 97. Am. Math. Soc., Providence (2002) 